\title{
Partitions and Objective Indefiniteness in Quantum Mechanics
}

\author{
David Ellerman \\ Department of Philosophy \\ U. of California/Riverside
}

October 4, 2018

\begin{abstract}
Classical physics and quantum physics suggest two meta-physical types of reality: the classical notion of a objectively definite reality with properties "all the way down," and the quantum notion of an objectively indefinite type of reality. The problem of interpreting quantum mechanics (QM) is essentially the problem of making sense out of an objectively indefinite reality. These two types of reality can be respectively associated with the two mathematical concepts of subsets and quotient sets (or partitions) which are category-theoretically dual to one another and which are developed in two mathematical logics, the usual Boolean logic of subsets and the more recent logic of partitions. Our sense-making strategy is "follow the math" by showing how the logic and mathematics of set partitions can be transported in a natural way to Hilbert spaces where it yields the mathematical machinery of QM-which shows that the mathematical framework of QM is a type of logical system over $\mathbb{C}$. And then we show how the machinery of QM can be transported the other way down to the set-like vector spaces over $\mathbb{Z}_{2}$ showing how the classical logical finite probability calculus (in a "non-commutative" version) is a type of "quantum mechanics" over $\mathbb{Z}_{2}$, i.e., over sets. In this way, we try to make sense out of objective indefiniteness and thus to interpret quantum mechanics.
\end{abstract}

\section{Contents}

1 Two types of reality 2

1.1 Objective definiteness and objective indefiniteness . . . . . . . . . . 2

$1.2 \quad$ Mathematical description of indefiniteness $=$ partitions $\ldots \ldots \ldots \ldots \ldots$

1.3 Mathematical description of definiteness $=$ subsets $\ldots \ldots \ldots \ldots \ldots$

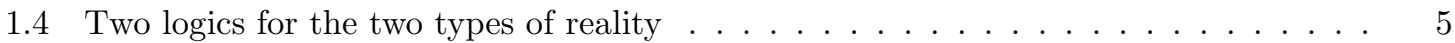

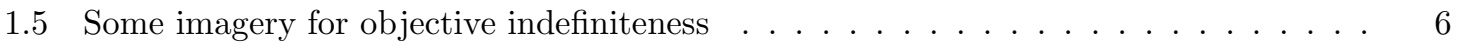

1.6 The two lattices . . . . . . . . . . . . . . . . . . . 8

2 Whence set partitions? 10

2.1 Set partitions from set attributes . . . . . . . . . . . . . . . . 10

2.2 Set partitions from set representations of groups $\ldots \ldots \ldots \ldots \ldots \ldots$

2.3 Set partitions from other set partitions . . . . . . . . . . . . . . . . . 11

3 Lifting partition concepts from sets to vector spaces 12

3.1 The basis principle . . . . . . . . . . . . . . . . . 12

3.2 What is a vector space partition? . . . . . . . . . . . . . . . . . 13

3.3 What is a vector space attribute? . . . . . . . . . . . . . 14 
4 Whence vector-space partitions? 14

4.1 Vector-space partitions from vector-space attributes . . . . . . . . . . . 14

4.2 Vector-space partitions from vector-space representations of groups . . . . . . . . . . 14

4.3 Vector-space partitions from other vector-space partitions . . . . . . . . . . . 16

5 Quantum Mechanics over sets 18

5.1 The pedagogical model of $\mathrm{QM}$ over $\mathbb{Z}_{2} \ldots \ldots \ldots \ldots$. . . . . . . . . . . 18

5.2 Vector spaces over $\mathbb{Z}_{2} \ldots \ldots . \ldots \ldots \ldots \ldots$

5.3 The brackets ............................ . . . 19

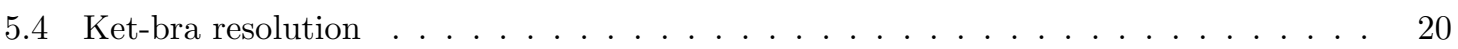

5.5 The norm ............................. . . . 20

5.6 The Born Rule . . . . . . . . . . . . . . . . . . . . . . . . . . . 20

5.7 Spectral decomposition on sets . . . . . . . . . . . . . . . . . . 21

5.8 Lifting and internalization . . . . . . . . . . . . . . . . . . . . . 21

5.9 Completeness and orthogonality of projection operators . . . . . . . . . . . . 22

5.10 Pythagorean Theorem for sets . . . . . . . . . . . . . . . . . . . . 22

5.11 Whence the Born Rule? . . . . . . . . . . . . . . . . . . . . . . . . . . 22

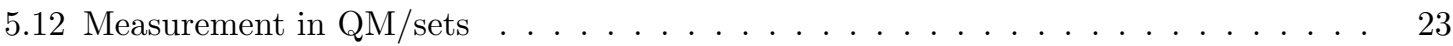

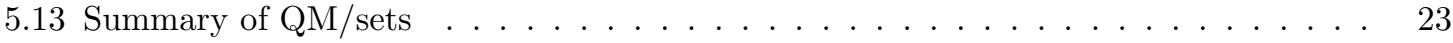

5.14 Whence von Neumann's Type 1 and Type 2 processes? . . . . . . . . . . . . . . 24

6 Final remarks

\section{Two types of reality}

\subsection{Objective definiteness and objective indefiniteness}

Our thesis in this paper is that mathematics (including logic) can be used to attack the problem of finding a realistic interpretation of (standard Dirac-von-Neumann) quantum mechanics (QM). Mathematics itself contains a very basic duality that can be associated with two meta-physical types of reality:

1. the common-sense notion of objectively definite reality assumed in classical physics, and

2. the notion of objectively indefinite reality suggested by quantum physics.

The problem of interpreting quantum mechanics is essentially the problem of making sense out of the notion of objective indefiniteness.

The approach taken here is to follow the lead of the mathematics of partitions, first for sets (where things are relatively "clear and distinct") and then for complex vector spaces where the mathematics of full QM resides.

There has long been the notion of subjective or epistemic indefiniteness ("cloud of ignorance") that is slowly cleared up with more discrimination and distinctions (as in the game of Twenty Questions). But the vision of reality that seems appropriate for quantum mechanics is objective or ontological indefiniteness. The notion of objective indefiniteness in QM has been most emphasized by Abner Shimony ([37, [38, [39]).

From these two basic ideas alone - indefiniteness and the superposition principle - it should be clear already that quantum mechanics conflicts sharply with common sense. If the quantum state of a system is a complete description of the system, then a quantity that has an indefinite value in that quantum state is objectively indefinite; its value is not merely unknown by the scientist who seeks to describe the system. [37, p. 47] 
The fact that in any pure quantum state there are physical quantities that are not assigned sharp values will then mean that there is objective indefiniteness of these quantities. [39, p. 27]

The view that a description of a superposition quantum state is a complete description means that the indefiniteness of a superposition state is in some sense objective or ontological and not just subjective or epistemic.

In addition to Shimony's "objective indefiniteness" (the phrase also used by Gregg Jaeger [24] and used here), other philosophers of physics have suggested related ideas such as:

- Peter Mittelstaedt's "incompletely determined" quantum states with "objective indeterminateness" 33,

- Paul Busch and Gregg Jaeger's "unsharp quantum reality" [5],

- Paul Feyerabend's "inherent indefiniteness" [18,

- Allen Stairs' "value indefiniteness" and "disjunctive facts" [40],

- E. J. Lowe's "vague identity" and "indeterminacy" that is "ontic" [29],

- Steven French and Decio Krause's "ontic vagueness" [21,

- Paul Teller's "relational holism" 41], and so forth.

Indeed, the idea that a quantum state is in some sense blurred or like a cloud is now rather commonplace even in the popular literature. Thus the idea of objective indefiniteness (described in various ways) is hardly new. Our goal is to give the mathematical backstory to indefiniteness by showing how the mathematical framework of QM can be built up or developed starting with the (new) mathematical logic appropriate for indefiniteness that is duality-related to the usual Boolean logic associated with classical definiteness.

\subsection{Mathematical description of indefiniteness = partitions}

How can indefiniteness be depicted mathematically? The basic idea is simple; start with what is taken as full definiteness and then factor or quotient out the surplus definiteness using an equivalence relation or partition.

Starting with some universe set $U$ of fully distinct and definite elements, a partition $\pi=\left\{B_{i}\right\}$ (i.e., a set of disjoint blocks $B_{i}$ that sum to $U$ ) collects together in a block (or cell) $B_{i}$ the distinct elements $u \in U$ whose distinctness is to be ignored or factored out, but the blocks are still distinct from each other. Each block represents the elements that are the same in some respect (since each block is an equivalence class in the associated equivalence relation on $U$ ), so the block is indefinite between the elements within it. But different blocks are still distinct from each other in that aspect.

Example 1 Consider the calculation of the binomial coefficient $\left(\begin{array}{l}N \\ m\end{array}\right)=\frac{N !}{m !(N-m) !}$. The idea is to count the number of $m$-ary subsets of an $N$-ary set $(m \leq N)$ where the different orderings of the otherwise same $m$-ary subset are surplus that need to be factored out. The method of calculation is to first count the number of possible orderings of the whole $N$-ary subset which is $N !=N(N-1) \ldots(2)(1)$. Then we want to quotient out the cases that are distinct only because of different orderings. For any given ordering of the $N$ elements, there are $m$ ! ways to permute the first $m$ elements in the given ordering-leaving the last $N-m$ elements the same. Thus we take the first quotient by identifying any two of the $N$ ! different orderings if they differ only in a permutation of those first $m$ elements. Since there are $m$ ! such permutations, there are now $N ! / m$ ! equivalence classes or blocks in the resulting 
partition of the $N$ ! orderings. But these equivalence classes still count as distinct the different orderings of the last $N-m$ elements so we further identify blocks which just have a permutation of the last $N-m$ elements to make larger blocks. Then the result is $\left(\begin{array}{l}N \\ m\end{array}\right)=\frac{N !}{m !(N-m) !}$ blocks in the partition which is the number of $m$-element subsets (which equals the number of $N-m$-element subsets) out of an $N$-ary set disregarding the ordering of the elements.

In this example, the set of fully determinate alternatives are the $N$ ! orderings of the $N$-element set. Then to consider the subsets of determinate or definite cardinality $m$ (and thus the complementary subsets of definite cardinality $N-m$ ), we must quotient out the number of possible orderings $m$ ! and $(N-m)$ ! to render the ordering of the elements in the subsets indefinite or indeterminate.

Example 2 To be concrete, consider a set $\{a, b, c, d\}$ of $N=4$ elements so the universe $U$ for fully distinct orderings has $4 !=24$ elements $\{a b c d, a b d c, \ldots\}$. How many 2 -element subsets are there? The first quotient groups together or identifies the orderings which only permute the first $m=2$ elements so two of the blocks in that partition are $\{a b c d, b a c d\}$ and $\{a b d c, b a d c\}$, and there are $N ! / m !=24 / 2=12$ such blocks. Each block has the same final $N-m=2$ elements in the ordering so we further identify the blocks that differ only in a permutation of those last $N-m$ elements. One of the blocks in that final partition is $\{a b c d, b a c d, a b d c, b a d c\}$ and there are $\frac{N !}{m !(N-m) !}=\frac{24}{(2)(2)}=6$ such blocks with four elements in each block. Each block is distinct from the other blocks in the first $m$ elements and in the last $N-m$ elements of the orderings in the block so the block count is just the number of subsets of $m$ elements (which equals the number of subsets of $N-m$ elements as well) where each block is indefinite as to the ordering of elements within the first $m$ elements and within the last $N-m$ elements.

Hermann Weyl makes the same point using an example slightly more complicated than the binomial coefficient. He starts with a set or "aggregate $S \ldots$ of elements each of which is in a definite state" [43, p. 239] and then considers a partition or equivalence relation whose $k$ blocks or classes $C_{1}, \ldots, C_{k}$ can be thought of as boxes into which the $n$ elements of $S$ may be placed (some boxes might be empty).

A definite individual state of the aggregate $S$ is then given if it is known, for each of the $n$ marks $p$ [DE: which distinguish the $n$ elements of $S$ ], to which of the $k$ classes [or boxes] the element marked $p$ belongs. Thus there are $k^{n}$ possible individual states of $S$. If, however, no artificial differences between elements are introduced by their labels $p$ and merely the intrinsic differences of state are made use of, then the aggregate is completely characterized by assigning to each class $C_{i}(i=1, \ldots, k)$ the number $n_{i}$ of elements of $S$ that belong to $C_{i}$. [43, p. 239]

Those occupation numbers $n_{i}$ would then characterize "the visible or effective state of the system S." [43, p. 239] Thus Weyl points out that the mathematical treatment of indefiniteness starts with the definiteness given here by the markings $p$ on the $n$ elements distributed between the $k$ boxes $C_{i}$, and then one erases the markings $p$ so the blocks or boxes in the partition have only an occupation number $n_{i}$ with no distinctions between the $n_{i}$ elements in each box $C_{i}$. When this scheme for representing indefiniteness is applied in quantum mechanics, then it is an objective indefiniteness in that no further differentiation between the elements of a box $C_{i}$ is possible.

Since photons come into being and disappear, are emitted and absorbed, they are individuals without identity. No specification beyond what was previously called the effective state of the aggregate is therefore possible. Hence the state of a photon gas is known when for each possible state $\alpha$ of a photon the number $n_{\alpha}$ of photons in that state is given (Bose-Einstein statistics of radiation). [43, p. 246] 
Thus within QM, the treatment of the indefiniteness due to the indistinguishability of quantum particles of the same type is to artificially treat them as distinct and then collect together or superpose the permutations of the particles (in a totally symmetric or totally antisymmetric manner) that factors out their supposed distinctness (see any QM text such as [9]).

Our point in this section is the general mathematical theme that indefiniteness is described by taking a partition or quotient of a set of definite entities. A partition is a mixture of indefiniteness and definiteness. Each block is indefinite between the elements within it, but the blocks of the partition are distinct from one another.

\subsection{Mathematical description of definiteness $=$ subsets}

The common-sense classical view of reality is that it is completely definite or determined and fully propertied all the way down. Every entity or thing definitely has a property $P$ or definitely has the property $\neg P$. Peter Mittelstaedt quotes Immanuel Kant's treatment of the idea of complete determinateness:

Every thing as regards its possibility is likewise subject to the principle of complete determination according to which if all possible predicates are taken together with the contradictory opposites, then one of each pair of contradictory opposites must belong to it. (Kant quoted in: [33, p. 170])

Given a universe set $U$, a predicate $P$ is represented by the subset $S \subseteq U$ of elements that have the property, and the complement subset $S^{c}=U-S$ represents the elements that have the property $\neg P$.

\subsection{Two logics for the two types of reality}

The two mathematical concepts of subsets and partitions are thus associated with two aforementioned metaphysical types of reality:

1. the common-sense notion of objectively definite reality assumed in classical physics, and

2. the notion of objectively indefinite reality suggested by quantum mechanics.

Subsets and quotient sets (or partitions) are mathematically dual concepts in the reverse-thearrows sense of category-theoretic duality, e.g., a subset is the direct image of a set monomorphism while a set partition is the inverse image of an epimorphism. This duality is familiar in abstract algebra in the interplay of subobjects (e.g., subgroups, subrings, etc.) and quotient objects. William Lawvere calls the general category-theoretic notion of a subobject a part, and then he notes: "The dual notion (obtained by reversing the arrows) of 'part' is the notion of partition." [28, p. 85]

The logic appropriate for the usual notion of fully definite reality described by subsets is the ordinary Boolean logic of subsets [3] (usually mis-specified as the special case of "propositional" logic). We have seen that the other vision of objectively indefinite reality suggested by QM is mathematically described by the equivalent concepts of quotient sets, partitions, or equivalence relations. The Boolean logic of subsets has an equally fundamental logic of the dual quotient sets, equivalence relations, or partitions ([12] and [16]) 1] The two logics of the dual concepts are associated with the two visions of reality.

Boole developed not only the logic of subsets, he defined a normalized counting measure on subsets of a finite universe set to yield the notion of logical finite probability theory. By making the

\footnotetext{
${ }^{1}$ The Boolean logic of subsets and the logic of partitions are equally fundamental in that they are the two logics that take (subsets of and partitions on) arbitrary universe sets in their semantics. Other logics that have a precise semantics, such as intuitionistic logic, have some additional structures, such as topologies, order relations, accessibility relations, etc. on the universe sets and thus those logics partake of the specific nature of those additional structures.
} 
same mathematical moves in the logic of partitions, i.e., by defining a normalized counting measure on the partitions on a finite universe set (where partitions are represented as the complement of the associated equivalence relations), one obtains a notion of logical entropy ([11] and 13]) that adds to the interpretation of QM mathematics 2

Since our topic is to better understand objective indefiniteness, and thus to better understand the reality described by QM, we will be following the math of partitional concepts. The development of the mathematical concepts from the logical level up to QM is based on the natural bridge between set concepts and vector-space concepts using the notion of a basis set. We will transport partitional concepts across that bridge in both directions. We will see that the mathematics and logic of set partitions can be lifted or transported to complex (inner product) vector spaces where it yields essentially the mathematical machinery of QM (of course, not the specifically physical postulates such as the Hamiltonian or the DeBroglie relationships). This shows that the mathematical framework of QM is a type of logical system developed using complex vector spaces. The vector space concepts of full QM can also be transported back to set-like vector spaces over $\mathbb{Z}_{2}$ to yield a pedagogical model of "quantum mechanics over sets" or QM/sets [15. The probability calculus of QM/sets is a noncommutative version of the usual Laplace-Boole logical finite probability theory. The traffic in both directions supports the idea of interpreting QM in terms of objective indefiniteness as illuminated by the logic and mathematics of partitions [14].

\subsection{Some imagery for objective indefiniteness}

In subset logic, each element of the universe set $U$ either definitely has or does not have a given property $P$ (represented as a subset of the universe). Moreover an element has properties all the way down so that two numerically distinct entities must differ by some property as in Leibniz's principle of the identity of indiscernibles. 27. Change takes place by the definite properties changing. For a hound to go from point $A$ to point $B$, there must be some trajectory of definite ground locations from $A$ to $B$.

In the logic of partitions, a partition $\pi=\left\{B_{i}\right\}$ is made up of disjoint blocks $B_{i}$ whose union is the universe set $U$ (the blocks are also thought of as the equivalence classes in the associated equivalence relation). The blocks in a partition have been distinguished from each other, but the elements within each block have not been distinguished from each other by that partition, i.e., they are identified by the associated equivalence relation. Hence each block can be viewed as the settheoretic version of a superposition of the distinct elements in the block. When more distinctions are made (the set-version of a measurement), the blocks get smaller and the partitions (set-version of mixed states) become more refined until the discrete partition $\mathbf{1}=\{\{u\}:\{u\} \subseteq U\}$ is reached where each block is a singleton (the set-version of a non-degenerate measurement yielding a completely decoherent mixed state). Change takes place by some attributes becoming more definite and other (incompatible) attributes becoming less definite. For a hawk, as opposed to a hound, to go from point $A$ to point $B$, it would go from a definite perch at $A$ into a flight of indefinite ground locations, and then would have a definite perch again at $B{ }^{3}$

\footnotetext{
${ }^{2}$ Although beyond the scope of this paper (see 15]), logical information theory gives the interpretation of the off-diagonal "coherences" in density matrices and the change in those off-diagonal entries following a measurement as well as the mathematical description of the non-unitary measurement process itself.

${ }^{3}$ The "flights and perchings" metaphor is from William James [25] p. 158] and according to Max Jammer, that description "was one of the major factors which influenced, wittingly or unwittingly, Bohr's formation of new conceptions in physics." [26, p. 178] The hawks and hounds pairing comes from Shakespeare's Sonnet 91.
} 


\begin{tabular}{|l|l|}
\hline $\begin{array}{l}\text { Classical trajectory from A to B. How a } \\
\text { hound goes from A to B. }\end{array}$ & $\begin{array}{l}\text { Subjective indefiniteness about classical } \\
\text { position ("cloud of ignorance"). }\end{array}$ \\
\hline $\begin{array}{l}\text { Objective indefiniteness of quantum } \\
\text { trajectory: definite position at A, } \\
\text { indefinite position in transition, and } \\
\text { definite position at B. How a hawk goes } \\
\text { from A to B. }\end{array}$
\end{tabular}

Figure 1: How a hound and a hawk go from $A$ to $B$

The imagery of having a sharp focus versus being out-of-focus could also be used if one is clear that it is the reality itself that is in-focus or out-of-focus, not just the image through, say, a microscope. A classical trajectory is like a moving picture of sharp or definite in-focus realities, whereas the quantum trajectory starts with a sharply focused reality, goes out of focus, and then returns to an in-focus reality (by a measurement).

In the objective indefiniteness interpretation, a subset $S \subseteq U$ of a universe set $U$ should be thought of as a single indefinite element $S$ that is only represented as a subset of fully definite elements $\{u: u \in S\}$-just as a single superposition vector is represented as a weighted vector sum of certain basis of eigen ("eigen" should be translated as "definite" here) vectors. Abner Shimony (37] and [38]), in his description of a superposition state as being objectively indefinite, sometimes used Heisenberg's 22 language of "potentiality" and "actuality" to describe the relationship of the eigenvectors that are superposed to give an objectively indefinite superposition. This terminology could be adapted to the case of the sets. The singletons $\{u\} \subseteq S$ are "potential" in the objectively indefinite superposition $S$, and, with further distinctions, the indefinite element $S$ might "actualize" to $\{u\}$ for one of the "potential" $\{u\} \subseteq S$. Starting with $S$, the other $\{u\} \nsubseteq S$ (i.e., $u \notin S$ ) are not "potentialities" that could be "actualized" with further distinctions.

This terminology is, however, somewhat misleading since the indefinite element $S$ is perfectly actual(in the objectively indefinite interpretation); it is only the multiple eigen-elements $\{u\} \subseteq S$ that are "potential" until "actualized" by some further distinctions. A non-degenerate measurement is not a process of a potential entity becoming an actual entity, it is a process of an actual indefinite element becomes an actual definite element. Since a distinction-creating measurement goes from actual indefinite to actual definite, the potential-to-actual language of Heisenberg should only be used with proper care-if at all.

Note that there are two conceptually distinct connotations for the mathematical subset $S \subseteq U$. In the classical interpretation, it is a set of fully definite elements of $u \in S$. In the quantum interpretation of a subset $S$, it is a single indefinite element that with further distinctions could become one of the singleton definite-elements or eigen-elements $\{u\} \subseteq S$.

Consider a three-element universe $U=\{a, b, c\}$ and a partition $\pi=\{\{a\},\{b, c\}\}$. The block $S=\{b, c\}$ is objectively indefinite between $\{b\}$ and $\{c\}$ so those singletons are its "potentialities" in the sense that a distinction could result in either $\{b\}$ or $\{c\}$ being "actualized." However $\{a\}$ is not a "potentiality" when one is starting with the indefinite element $\{b, c\}$.

Note that this objective indefiniteness of $\{b, c\}$ is not well-described as saying that indefinite pre-distinction element is "simultaneously both $b$ and $c$ " (like the common misdescription of the undetected particle "going through both slits" in the double-slit experiment); instead it is indefinite 
between $b$ and $c$. That is, a superposition of two sharp eigen-alternatives should not be thought of like a double-exposure photograph which has two fully definite images (e.g., simultaneously a picture of say $b$ and $c$ ). Instead of a double-exposure photograph, the superposition should be thought of as representing a blurred or incomplete reality that with further distinctions could sharpen to either of the sharp realities. But there must be some way to indicate which sharp realities could be obtained by making further distinctions (measurements), and that is why the blurred or cloud-like indefinite reality is represented by mathematically superposing the sharp potentialities.

This point might be illustrated using some Guy Fawkes masks.

\begin{tabular}{|l|}
\hline $\begin{array}{l}\text { Eigenstate 1: } \\
\text { Guy Fawkes with goatee }\end{array}$ \\
\hline $\begin{array}{l}\text { Eigenstate 2: } \\
\text { Guy Fawkes with mustache }\end{array}$ \\
\hline $\begin{array}{l}\text { Objectively indistinct state before } \\
\text { (facial hair) distinctions were } \\
\text { made is the pre-distinction state. }\end{array}$ \\
\hline $\begin{array}{l}\text { But that objectively indistinct } \\
\text { state may be represented by } \\
\text { superposition of possible distinct } \\
\text { alternatives, the set } \\
\text { \{goatee, mustache\} } \\
\text { or vector } \\
\text { (goatee }\rangle+ \text { mustache }\rangle\end{array}$ \\
\hline
\end{tabular}

Figure 2: Objectively indefinite pure state represented as superposition of distinct eigen-alternatives

Instead of a double-exposure photograph, a superposition representation might be thought of as "a photograph of clouds or patches of fog." (Schrödinger quoted in: [20, p. 66]) Schrödinger distinguishes a "photograph of clouds" from a blurry photograph presumably because the latter might imply that it was only the photograph that was blurry while the underlying objective reality was sharp. The "photograph of clouds" imagery for a superposition connotes a clear and complete photograph of an objectively "cloudy" or indefinite reality. Regardless of the (imperfect) imagery, one needs some way to indicate what are the definite eigen-elements that could be "actualized" from a single indefinite element $S$, and that is the role in the set case of conceptualizing a subset $S$ as a collecting together or "superposing" certain "potential" eigen-states, i.e., the singletons $\{u\} \subseteq S$.

\subsection{The two lattices}

The two dual subset and partition logics are modeled by the two lattices (or, with more operations, algebras) of subsets and of partitions 4 The conceptual duality between the lattice of subsets (the lattice part of the Boolean algebra of subsets of $U$ ) and the lattice of partitions could be described (again following Heisenberg) using the rather meta-physical notions of substance5 and form (as

\footnotetext{
${ }^{4}$ The two logics and lattices are "dual" or "duality-related" in the sense of the subset-partition duality.

${ }^{5}$ Heisenberg identifies "substance" with energy.

Energy is in fact the substance from which all elementary particles, all atoms and therefore all things are made, and energy is that which moves. Energy is a substance, since its total amount does not change, and the elementary particles can actually be made from this substance as is seen in many experiments on the creation of elementary particles. 22, p. 63]
} 
in in-form-ation)-which might be compared to the terms "matter" or "objects" on one hand and "structure" on the other hand in some modern metaphysical discussions 6

For each lattice where $U=\{a, b, c\}$, start at the bottom and move towards the top.

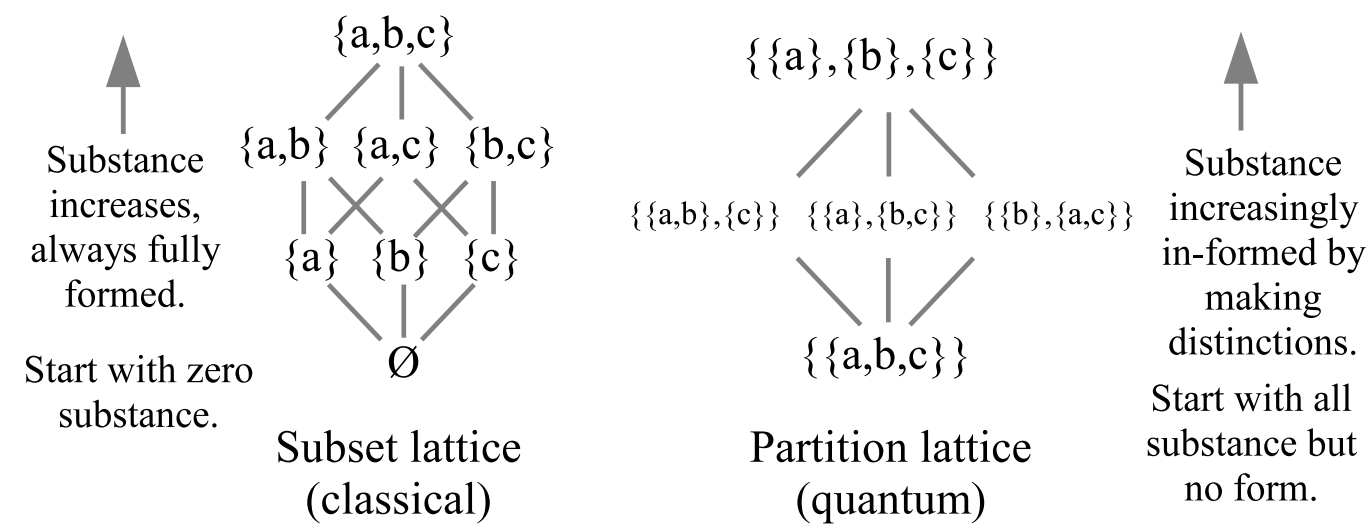

Figure 3: Conceptual duality between the subset and partition logics

At the bottom of the Boolean lattice is the empty set $\emptyset$ which represents no substance. As one moves up the lattice, new elements of substance always with fully definite properties are created until finally one reaches the top, the universe $U$. Thus new substance is created in moving up the lattice but each element is fully formed and distinguished in terms of its properties.

At the bottom of the partition lattice is the indiscrete partition or "blob" $\mathbf{0}=\{U\}$ (where the universe set $U$ makes one block) which represents all the substance but with no distinctions to in-form the substance 7 As one moves up the lattice, no new substance is created but distinctions objectively in-form the indistinct elements as they become more and more distinct, until one finally reaches the top, the discrete partition 1, where all the eigen-elements of $U$ have been fully distinguished from each other 8 It was previously noted that a partition combines indefiniteness (within blocks) and definiteness (between blocks). At the top of the partition lattice, the discrete partition $\mathbf{1}=$ $\{\{u\}:\{u\} \subseteq U\}$ is the result making all the distinctions to eliminate the indefiniteness. Thus one ends up at the "same" place (macro-universe of distinguished elements) either way, but by two totally different but dual ways 9

The progress from bottom to top of the two lattices could also be described as two creation stories.

- Subset creation story: "In the Beginning was the Void", and then elements are created, fully propertied and distinguished from one another, until finally reaching all the elements of the universe set $U$.

- Partition creation story: "In the Beginning was the Blob", which is an undifferentiated "substance," and then there is a "Big Bang" where elements ("its") are created by the substance being objectively in-formed (objectified information) by the making of distinctions (e.g., breaking symmetries) until the result is finally the singletons which designate the elements of the universe $U$.

\footnotetext{
${ }^{6}$ See McKenzie 32 and the references therein to ontic structural realism.

${ }^{7}$ The "blob" is the set-version of a pure state in QM prior to a distinctions-creating measurement that decoheres the pure state into non-blob partitions analogous to a mixed state (see [15] for spelling this out using density matrices).

${ }^{8}$ This notion of logical in-formation as distinctions is based on partition logic just as logical probability is based on subset logic ([1] and [13]). That is, the logical entropy of a partition is the normalized counting measure of the distinctions of a partition (represented as a binary relation) just as the Laplace-Boole logical probability of a subset is the normalized counting measure on the subsets (events) of the finite universe set (set of equiprobable outcomes).

${ }^{9}$ In treating the universe $U=\left\{u, u^{\prime}, \ldots\right\}$ and the discrete partition $\mathbf{1}=\left\{\{u\},\left\{u^{\prime}\right\}, \ldots\right\}$ as the "same" we are neglecting the distinction between $u$ and $\{u\}$ for $u \in U$.
} 
These two creation stories might also be illustrated as follows.

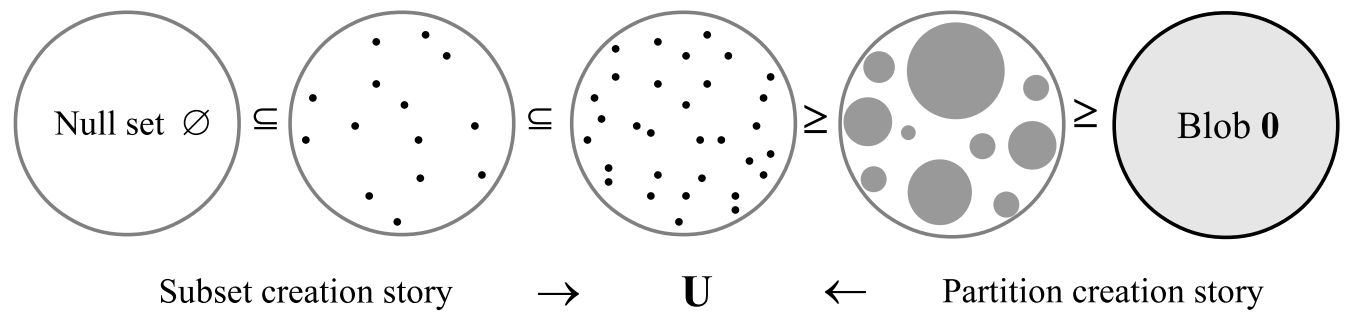

Figure 4: Two creation stories

One might think of the universe $U$ (in the middle of the above picture) as the macroscopic world of definite entities that we ordinarily experience. Common sense and classical physics assumes, as it were, the subset creation story on the left. But a priori, it could just as well have been the dual story, the partition creation story pictured on the right, that leads to the same macro-picture $U$.

Since partitions are the mathematical expression of indefiniteness, our strategy is to first show where set partitions come from and then to "lift" or "transport" the partitional machinery to vector spaces using the notion of a basis set of a vector space. The result is essentially the mathematical machinery of quantum mechanics-all of which shows how quantum mechanics can be interpreted using the objective indefiniteness conception of reality that is associated at the logical level with partition logic.

\section{Whence set partitions?}

\subsection{Set partitions from set attributes}

Take the universe set as some specific set of people, say, in a room. People have numerical attributes like weight, height, or age as well as non-numerical attributes with other values such place of birth, family name, and country of citizenship. Abstractly an attribute on a universe set $U$ is a function $f: U \rightarrow R$ from $U$ to some set of values $R$ (usually the reals $\mathbb{R}$ ). In subset logic, an element $u \in U$ either has a property represented by a subset $S \subseteq U$ or not; in partition logic, an attribute $f$ assigns a value $f(u)$ to each $\{u\} \subseteq U$. The two concepts of a property and an attribute overlap for binary attributes where the attribute might be represented by the characteristic function $\chi_{S}: U \rightarrow 2=$ $\{0,1\}$ of a subset $S \subseteq U 10$

Each attribute $f: U \rightarrow R$ on a universe $U$ determines the inverse-image partition $f^{-1}=$ $\left\{f^{-1}(r) \neq \emptyset: r \in R\right\}$. Attributes are one way to define a partition on a set $U$. Since this method of defining a partition starts with a numerical attribute $f(u)$ already assigned to each $u \in U$, it may be called the top-down method.

\subsection{Set partitions from set representations of groups}

Another more "bottom-up" way to define a partition on $U$ is to map the elements $u \in U$ to "similar" (i.e., same block) elements $u^{\prime}$ by some set of transformations $G=\{t: U \rightarrow U\}$. This defines a binary relation: $u G u^{\prime}$ if there exists a $t \in G$ such that $t(u)=u^{\prime}$. In order to define a partition, the binary relation $u G u^{\prime}$ has to be an equivalence relation so the blocks of the partition are the equivalence classes. The three requirements for an equivalence relation are reflexivity, symmetry, and transitivity.

\footnotetext{
${ }^{10}$ To be technically precise, a subset $S$ is given by a binary attribute $\chi_{S}: U \rightarrow 2=\{0,1\}$ plus the designation of an element $1 \in 2$ so that $S=\chi_{S}^{-1}(1)$ as in Lawvere's well-known subobject-classifier diagram [28 p. 39].
} 
- For the relation to be reflexive, i.e., $u G u$ for all $u \in U$, it is sufficient for the set of transformations $G$ to contain the identity transformation $1_{U}: U \rightarrow U$.

- For the relation to be symmetric, i.e., $u G u^{\prime}$ implies $u^{\prime} G u$, it is sufficient for each $t \in G$ to have an inverse $t^{-1} \in G$ where $U \stackrel{t}{\longrightarrow} U \stackrel{t^{-1}}{\longrightarrow} U=1_{U}=U \stackrel{t^{-1}}{\longrightarrow} U \stackrel{t}{\longrightarrow} U$.

- For the relation to be transitive, i.e., $u G u^{\prime}$ and $u^{\prime} G u^{\prime \prime}$ imply $u G u^{\prime \prime}$, it is sufficient for each $t, t^{\prime} \in G$ that $t^{\prime} t: U \stackrel{t}{\longrightarrow} U \stackrel{t^{\prime}}{\longrightarrow} U$ is also in $G$.

These three conditions, the existence of the identity, the existence of an inverse, and closure under composition, define a transformation group $G=\{t: U \rightarrow U\}$, i.e., a group action on a set $U$. Equivalently, a set representation of a group $G$ is given by a group homomorphism $T: G \rightarrow S(U)$, where $S(U)$ is the symmetric group of permutations $t$ of the set $U$ (and where the transformation group $\{t: U \rightarrow U\} \subseteq S(U)$ is the image of the map). An abstract group satisfies these three conditions where the composition is also required to be associative in the sense that for any $t, t^{\prime}, t^{\prime \prime} \in G$, $\left(t^{\prime \prime} t^{\prime}\right) t=t^{\prime \prime}\left(t^{\prime} t\right)$. For a transformation group, the composition is automatically associative.

This connection between groups and equivalence relations or partitions is well-known, e.g., [ $\underline{6}$, and is probably as old as the notion of a group. Instead of elements $u, u^{\prime} \in U$ being collected in the same block by have the same attribute value $f(u)=f\left(u^{\prime}\right)$, the group transformations take any element $u$ to a "similar" or "symmetric" element $t(u)=u^{\prime}$. A subset $S \subseteq U$ is invariant under $G$ if for any $t \in G, t(S) \subseteq S$. A minimal invariant subset is an orbit, and the partition defined by the transformation group $G$ is the set partition of orbits. That is the bottom-up method of defining a set partition since we don't begin with some attribute-value already assigned to the elements of $U$.

What is the significance of the blocks in the partition of minimal invariant subsets? Often the treatment of symmetry groups focuses on what is invariant or conserved, e.g., the perspective of Noether's theorem [4].

There is another perspective with which to view the representations of symmetry groups. To represent an indefinite reality, there is first some notion of the fully definite eigen-alternatives that are then collected together or superposed to represent something indefinite between those alternatives.

\section{What determines the set of definite eigen-alternatives?}

Given a set of symmetries on a set, in what different ways can there be distinct subsets that still satisfy the constraints of the symmetry operations? The minimal invariant subsets or orbits of a set representation of a symmetry group provide the answer to that question about the variety of "atomic" eigen-forms consistent with the symmetries.

This question and the answer become more significant when we move beyond structure-less sets to linear vector spaces. The minimal invariant subsets, the orbits, then become the minimal invariant subspaces, the irreducible subspaces, which are the carriers of the irreducible representations or irreps in vector space representations of groups.

\subsection{Set partitions from other set partitions}

The notion of distinguishability (in principle, making a distinction) has long been recognized as fundamental in QM.

If you could, in principle, distinguish the alternative final states (even though you do not bother to do so), the total, final probability is obtained by calculating the probability for each state (not the amplitude) and then adding them together. If you cannot distinguish the final states even in principle, then the probability amplitudes must be summed before taking the absolute square to find the actual probability. [19, 3-9] 
In the foregoing, we have frequently referred to the making of distinctions as the set version of a measurement 11 What is the mathematical operation for making the distinctions (as in a measurement)? It is the join operation from partition logic. But before two set partitions can be joined to form a more refined partition with more distinctions, they must be compatible in the sense of being defined on the same universe set. If two set partitions $\pi=\{B\}$ and $\sigma=\{C\}$ are compatible, i.e., are partitions of the same universe $U$, then their join $\pi \vee \sigma$ is the set partition whose blocks are the non-empty intersections $B \cap C$.

Since two set attributes $f: U \rightarrow \mathbb{R}$ and $g: U^{\prime} \rightarrow \mathbb{R}$ define two inverse image partitions $\left\{f^{-1}(r)\right\}$ and $\left\{g^{-1}(s)\right\}$ on their domains, we need to extend the concept of compatible partitions to the attributes that define the partitions. That is, two attributes $f: U \rightarrow \mathbb{R}$ and $g: U^{\prime} \rightarrow \mathbb{R}$ are compatible if they have the same domain $U=U^{\prime}$.

Given two compatible set attributes $f: U \rightarrow \mathbb{R}$ and $g: U \rightarrow \mathbb{R}$, the join of their eigenspace partitions has as blocks the non-empty intersections $f^{-1}(r) \cap g^{-1}(s)$. Each block in the join of the eigenspace partitions could be characterized by the ordered pair of eigenvalues $(r, s)$. An eigenvector of both $f, S \subseteq f^{-1}(r)$, and of $g, S \subseteq g^{-1}(s)$, would be a simultaneous eigenvector: $S \subseteq f^{-1}(r) \cap$ $g^{-1}(s)$.

A set of compatible set attributes is said to be complete if the join of their partitions is discrete, i.e., the blocks have cardinality 1. A Complete Set of Compatible Attributes or CSCA characterizes the singletons $\{u\} \subseteq U$ by the ordered $n$-tuple $(r, \ldots, s)$ of attribute values.

All this machinery of set partitions can be lifted or transported to vector spaces to give the mathematical machinery of QM 12

\section{Lifting partition concepts from sets to vector spaces}

\subsection{The basis principle}

There is a natural part-of-the-folklore bridge or ladder connecting set concepts to vector-space concepts. The basic idea is that a vector $v=\sum_{i} \alpha_{i} b_{i}$, represented in terms of a set $\left\{b_{i}\right\}$ of basis vectors, is a $K$-valued set where each element $b_{i}$ in the basis set takes a value $c_{i}$ in the base field $K$. Given a set concept, the basis principle is that one can generate the corresponding vector-space concept by applying the set concept to a basis set and seeing what it generates. Starting with the set concept of cardinality, one arrives at the corresponding vector-space concept by applying the set concept to a basis set to arrive at the cardinality of the basis set. After checking that all bases have the same cardinality, this yields the vector-space notion of dimension. Thus the cardinality of a set lifts not to the cardinality of a vector space but to its dimension.

Some of the lifting is accomplished by the free vector space functor from the category of sets to the category of vector spaces over a given field $K$. A set $U$ is carried by this functor to the vector space $K^{U}$ spanned by the Kronecker delta basis $\left\{\delta_{u}: U \rightarrow K\right\}_{u \in U}$ where $\delta_{u}\left(u^{\prime}\right)=0$ for $u^{\prime} \neq u$ and $\delta_{u}(u)=1$. A set $U$ of a certain cardinality thus generates a vector space $K^{U}$ of the same dimension.

\footnotetext{
${ }^{11}$ Technically, a "distinction" of a partition $\pi=\{B\}$ on $U$ is an ordered pair $\left(u, u^{\prime}\right)$ of elements of $U$ in different blocks of the partition. The set of distinctions, dit $(\pi)$, of a partition $\pi$ is called a partition relation (or apartness relation in computer science) and is just the complement of the associated equivalence relation. The notion of a distinction of a partition is the partition logic analogue of an element of a subset in subset logic. For instance, given two partitions $\pi=\{B\}$ and $\sigma=\{C\}$ on a universe set and two subsets $S$ and $T$ of a universe set, the partition join $\pi \vee \sigma$ combines the distinctions of the partitions, i.e., $\operatorname{dit}(\pi \vee \sigma)=\operatorname{dit}(\pi) \cup \operatorname{dit}(\sigma)$, just as the subset join or union $S \cup T$ combines the elements of the subsets (see [12] or [16] for further developments).

${ }^{12}$ In QM, the extension of concepts on finite dimensional Hilbert space to infinite dimensional ones is well-known. Since our expository purpose is conceptual rather than mathematical, we will stick to finite dimensional spaces.
} 


\subsection{What is a vector space partition?}

A partition $\pi=\{B\}$ on a set $U$ is a set of subsets whose direct sum (i.e., disjoint union) is the whole set, i.e., a direct sum decomposition of the set. The corresponding vector space concept is a set of subspaces of a vector space whose direct sum is the vector space, i.e., a direct sum decomposition of the vector space. In terms of the basis principle, we could apply the set partition $\pi=\{B\}$ of a set $U$ to a basis set $\left\{b_{u}\right\}_{u \in U}$, then each block $B$ generates a subspace $V_{B}$ and the set of subspaces $\left\{V_{B}\right\}_{B \in \pi}$ is a direct sum decomposition of the vector space spanned by the basis set. Thus the lift or transport of the concept of a set partition is a direct sum decomposition of a vector space. In particular, it is not a set partition of a vector space that is compatible with the vector space operations, i.e., a quotient space $V / W$ as would be defined by each subspace $W \subseteq V$ with the equivalence relation $v \sim v^{\prime}$ if $v-v^{\prime} \in W$. While a partition on a set is essentially the same as a quotient set (or equivalence relation on the set), the vector-space lift of a set partition is not a quotient vector space but a direct sum decomposition of a vector space. In lifting or transporting the partitional concepts for sets to vector spaces, we are making the choices guided by the set-to-basis-set connection which yields the mathematical machinery of quantum mechanics.

This is not particularly new; it is part of the mathematical folklore. Hermann Weyl outlined the lifting program by first considering an attribute on a set, which defined the set partition or "grating" [43. p. 255] of elements with the same attribute-value. Then he moved to the quantum case where the set or "aggregate of $n$ states has to be replaced by an $n$-dimensional Euclidean vector space" [43. p. 256] 13 The appropriate notion of a partition or "grating" is a "splitting of the total vector space into mutually orthogonal subspaces" so that "each vector $\vec{x}$ splits into $r$ component vectors lying in the several subspaces" [43, p. 256], i.e., a direct sum decomposition of the space, where the subspaces are the eigenspaces of an observable operator.

Weyl's grating metaphor also lends itself to (our own example of) seeing measurement of the, say, 'regular polygonal shape' of an 'indefinite blob of dough' as it randomly falls through a opening in a grating to take on that 'polygonal shape' (with the attribute-value or eigenvalue being the number of regular sides $\lambda=3,4,5,6)$.

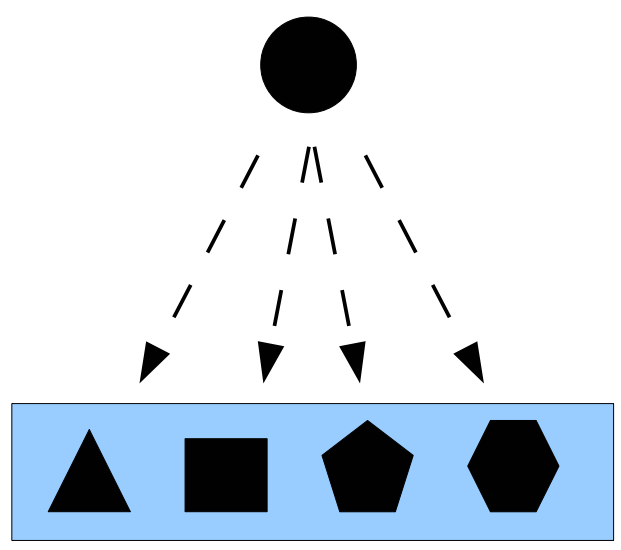

Figure 5: Imagery of measurement as randomly giving an indefinite blob of dough a definite eigen-shape.

Note how the blob of dough is objectively indefinite between the regular polygonal shapes and does not simultaneously have all those shapes even though it might be mathematically represented as the set $\{\boldsymbol{\Lambda}, \ldots\}$ or the superposition vector $\boldsymbol{\Lambda}+\boldsymbol{\square}+\ldots$ in a certain space.

\footnotetext{
${ }^{13}$ Note the lift from sets to vector spaces using the basis principle where the cardinality $n$ becomes dimension $n$.
} 


\subsection{What is a vector space attribute?}

A set attribute is a function $f: U \rightarrow \mathbb{R}$ (where the set of values is taken as the reals). The inverseimage $f^{-1}(r) \subseteq U$ of each value $f(u)=r$ is a subset where the attribute has the same value, and those subsets form a set partition. Given a basis set $\left\{b_{u}\right\}_{u \in U}$ of a vector space $V$ over a field $K$, we can apply a set attribute $f:\left\{b_{u}\right\}_{u \in U} \rightarrow K$ to the basis set and see what it generates. One possibility is to linearly extend the function $f^{*}\left(b_{u}\right)=f\left(b_{u}\right)$ to the whole space to obtain a linear functional $f^{*}: V \rightarrow K$. But a linear functional defines a quotient space $V / f^{*-1}(0)$, not a vector space partition.

The same information $f:\left\{b_{u}\right\}_{u \in U} \rightarrow K$ also defines $\hat{f}\left(b_{u}\right)=f\left(b_{u}\right) b_{u}$ which linearly extends to a linear operator $\hat{f}: V \rightarrow V$. The given basis vectors $\left\{b_{u}\right\}$ are eigenvectors of the operator $\hat{f}$ with the eigenvalues $f\left(b_{u}\right)$, and the eigenspaces are the subspaces where the operator has the same eigenvalue. The eigenvectors span the whole space so we see that the lift or transport of a set attribute, which defines a set partition, is a vector space linear operator whose eigenspaces are a vector space partition (i.e., direct sum decomposition) of the whole space, i.e., a diagonalizable linear operator 14

\section{Whence vector-space partitions?}

\subsection{Vector-space partitions from vector-space attributes}

Given a diagonalizable linear operator $L: V \rightarrow V$, where $V$ is a finite-dimensional vector space over a field $K$ and where $\lambda_{1}, \ldots, \lambda_{k}$ are the distinct eigenvalues, then there are projection operators $P_{i}$ for $i=1, \ldots, k$ such that:

1. $L=\sum_{i=1}^{k} \lambda_{i} P_{i}$;

2. $I=\sum_{i=1}^{k} P_{i}$;

3. $P_{i} P_{j}=0$ for $i \neq j$; and

4. the range of $P_{i}$ is the eigenspace $V_{i}$ for the eigenvalue $\lambda_{i}$ for $i=1, \ldots, k$. 23, Theorem 8, p. $172]$

What is the vector space partition canonically defined by a diagonalizable linear operator? Any basis of eigenvectors could be seen as defining a direct sum of the one-dimensional subspaces spanned by those eigenvectors. But those subspaces are far from unique. But if we group together all the eigenvectors with the same eigenvalue (i.e., use the top-down method to define a vector-space partition), then they span the eigenspaces. It is the set of eigenspaces $\left\{V_{i}\right\}$ that gives the unique canonical direct-sum decomposition or vector-space partition defined by a (diagonalizable) linear operator. This standard linear algebra result holds for any base field, but for QM, the base field is the complex numbers $\mathbb{C}$. In order for the eigenvalues to always be real, the diagonalizable linear operators are required to be Hermitian (or self-adjoint, i.e., equal to their conjugate transposes).

\subsection{Vector-space partitions from vector-space representations of groups}

A vector-space representation of an abstract group $G$ is a group homomorphism $T: G \rightarrow G L(V)$ where $G L(V)$ is the group of invertible linear transformations $V \rightarrow V$ of a vector space $V$ over the complex numbers. Here again, the idea is to define a (vector-space) partition by a (linear) group of transformations $T_{g}: V \rightarrow V$ that map elements $v \in V$ to similar or symmetric elements $T_{g}(v)$. A

\footnotetext{
${ }^{14} \mathrm{~A}$ diagonalizable linear operator is the lift of a set attribute $f$ that is a total function, so a non-diagonalizable linear operator is the lift of a partial function $f$.
} 
subspace $W \subseteq V$ is invariant if $T_{g}(W) \subseteq W$ for all $g \in G$. And again, it is the minimal invariant subspaces, the irreducible subspaces, that are of interest. The irreducible subspaces $\left\{W_{\alpha}\right\}$ are the carriers for the irreducible representations $T \uparrow: W_{\alpha} \rightarrow W_{\alpha}$ or irreps. And the representation space $V$ is a direct sum of some set of irreducible subspaces $V=\sum_{i=1}^{l} \oplus W_{i}$ so the vector-space representation of a group defines a vector-space partition of the space. But these vector-space partitions are not unique and are thus not canonically defined by the representation.

Finding such a decomposition [of irreps] is an exact analogue of finding a basis of eigenvectors of a single operator. In neither case is the decomposition unique. However, in the operator case the eigenvalues and the multiplicities of occurrence are uniquely determined. Moreover the linear span of these basis vectors with a common eigenvalue is just the total eigenspace for that eigenvalue and is uniquely determined. The decomposition as a direct sum of eigenspaces is unique. [30, p. 244]

Hence the problem in this bottom-up approach is "finding an analogue for equality of eigenvalues" [30, p. 244] to group the irreps together.

Suppose $T$ is a representation of $G$ acting on a space $V$ and $T^{\prime}$ is a representation of the same $G$ acting on a space $V^{\prime}$. Then a linear map $\phi: V \rightarrow V^{\prime}$ is said morphism of representations or intertwining map if for all $g \in G$ and all $v \in V$ :

$$
\begin{gathered}
\phi\left(T_{g}(v)\right)=T_{g}^{\prime}(\phi(v)), \text { i.e., } \\
V \quad \stackrel{T_{g}}{\longrightarrow} \quad V \\
\phi \downarrow \\
V^{\prime} \stackrel{T_{g}^{\prime}}{\longrightarrow} \quad V^{\prime} \\
\text { commutes. }
\end{gathered}
$$

If $\phi$ is also invertible, then $\phi$ is said to be an isomorphism of representations, and the representations are said to be isomorphic or equivalent.

The remarkable fact is that each group has a fixed set of inequivalent irreps, so the distinct irreps are a characteristic of the group itself, not of a particular representation.

The uniqueness and canonical nature of the partition obtained in the operator case by equality of eigenvalues is now obtained using equivalence of irreps and their underlying irreducible subspaces. All the irreducible subspaces $W_{i}$ for irreps equivalent to an irrep $L$ in any such direct sum $V=\sum_{i=1}^{l} \oplus W_{i}$ are grouped together (by direct sum) to obtain the invariant carrier $W_{L}$ for a primary representationwhere a representation is primary if all its irreducible subrepresentations are equivalent and the underlying carrier space is also called primary. Note that some of the inequivalent irreps of the group $G$ may not be involved in a particular representation. The decomposition of $V$ as the direct sum $\sum_{L} \oplus W_{L}$ of the invariant primary subspaces for the primary representations is unique. "It is the invariant subspaces $\left[W_{L}\right]$ which are the analogues of the eigenspaces of a single operator." [30, p. 244] In terms of representations rather than their carrier subspaces, it is the unique "canonical decomposition into primary representations." [30, p. 244]

Thus we have the top-down construction of the vector space partition $V=\sum \oplus V_{i}$ of eigenspaces $V_{i}$ given by an operator (or vector-space attribute) and the bottom-up construction of the vectorspace partition $V=\sum \oplus W_{L}$ of the carriers $W_{L}$ for primary representations given by a vector-space representation of a symmetry group.

The following table brings out the analogies between the top-down and bottom-up determination of vector-space partitions. 


\begin{tabular}{|l|l|l|}
\cline { 2 - 3 } \multicolumn{1}{c|}{$\begin{array}{c}\text { Top-down and } \\
\text { Bottom-up }\end{array}$} & $\begin{array}{l}\text { Top-down determination of } \\
\text { partition by an operator }\end{array}$ & $\begin{array}{l}\text { Bottom-up determination of } \\
\text { partition by group representation }\end{array}$ \\
\hline Eigen-alternatives & Eigenvectors & Irreducible represenations \\
\hline $\begin{array}{l}\text { Non-unique } \\
\text { partition }\end{array}$ & $\begin{array}{l}\text { Basis of eigenvectors gives } \\
\text { direct sum generated spaces }\end{array}$ & Direct sum of irreps \\
\hline $\begin{array}{l}\text { Criterion for } \\
\text { collecting }\end{array}$ & $\begin{array}{l}\text { Collecting eigenvectors by } \\
\text { eigenvalues }\end{array}$ & Collecting irreps by equivalence \\
\hline $\begin{array}{l}\text { Resulting } \\
\text { subspaces }\end{array}$ & Eigenspaces & Primary subspaces \\
\hline $\begin{array}{l}\text { Canonical vector- } \\
\text { space partitions }\end{array}$ & $\begin{array}{l}\text { Unique vector-space } \\
\text { partition of eigenspaces. }\end{array}$ & $\begin{array}{l}\text { Unique vector-space partition of } \\
\text { primary subspaces }\end{array}$ \\
\hline
\end{tabular}

Figure 6: Analogies between top-down and bottom-up determination of partitions

To represent indefiniteness, we first need to specify the "universe" of fully definite eigen-alternatives, and then indefiniteness can be described by collecting together or superposing the "potential" eigenalternatives. In the vector-space case, the eigen-alternatives determined by an operator are the eigenvectors and the eigen-alternatives determined by a representation of a symmetry group are the minimal invariant subspaces that are the carriers for the irreducible representations of the symmetry group.

For state-dependent (or extrinsic) attributes of a quantum particle like the linear momentum or angular momentum, the fully definite eigenstates are determined by the irreducible representations of the linear-translation or rotational-translation symmetry groups respectively. For the stateindependent (or intrinsic) attributes of quantum particles, like the mass, charge, and spin of an electron, they are determined in particle physics by the irreducible representations of the appropriate symmetry groups 15

\subsection{Vector-space partitions from other vector-space partitions}

The set notion of compatibility lifts to vector spaces, via the basis principle, by defining two vector space partitions $\omega=\left\{W_{\lambda}\right\}$ and $\xi=\left\{X_{\mu}\right\}$ on $V$ as being compatible if there is a basis set for $V$ so that the two vector space partitions are generated by two set partitions of that common or simultaneous basis set.

If two vector space partitions $\omega=\left\{W_{\lambda}\right\}$ and $\xi=\left\{X_{\mu}\right\}$ are compatible, then their vector space join $\omega \vee \xi$ is defined as the vector space partition whose subspaces are the non-zero intersections $W_{\lambda} \cap X_{\mu}$. And by the definition of compatibility, we could also generate the subspaces of the join $\omega \vee \xi$ by the blocks in the set join of the two set partitions of the common basis set.

Since real-valued set attributes lift to Hermitian linear operators, the notion of compatible set attributes just defined would lift to two linear operators being compatible if their eigenspace partitions are compatible. It is a standard fact of linear algebra [23, p. 177] that two diagonalizable linear operators $L, M: V \rightarrow V$ (on a finite dimensional space $V$ ) are compatible (i.e., have a basis of simultaneous eigenvectors) if and only if they commute, $L M=M L$. Hence the commutativity of linear operators is the lift of the compatibility (i.e., defined on the same set) of set attributes. That explains the importance of the notion of commutativity in QM and that is why the repeated compatible measurements, described mathematically as the join operation, requires commutativity. The join of two operator-eigenspace partitions is defined iff the operators commute. As Weyl put it:

\footnotetext{
${ }^{15}$ The classic paper in this group-theoretic treatment of particles is Wigner 44 . For recent overviews, see the group-theoretical definition of particles in Falkenburg [17] or Roberts 34.
} 
"Thus combination [DE: join] of two gratings [eigenspace partitions of two operators] presupposes commutability...". [43, p. 257]

Two commuting Hermitian linear operators $L$ and $M$ have compatible eigenspace partitions $W_{L}=\left\{W_{\lambda}\right\}$ (for the eigenvalues $\lambda$ of $L$ ) and $W_{M}=\left\{W_{\mu}\right\}$ (for the eigenvalues $\mu$ of $M$ ). The blocks in the join $W_{L} \vee W_{M}$ of the two compatible eigenspace partitions are the non-zero subspaces $\left\{W_{\lambda} \cap W_{\mu}\right\}$ which can be characterized by the ordered pairs of eigenvalues $(\lambda, \mu)$. The nonzero vectors $v \in W_{\lambda} \cap W_{\mu}$ are simultaneous eigenvectors for the two commuting operators, and there is a basis for the space consisting of simultaneous eigenvectors 16

A set of commuting linear operators is said to be complete if the join of their eigenspace partitions is nondegenerate, i.e., the blocks have dimension 1 . The join operation gives the results of compatible measurements so the join of a complete set of compatible vector space attributes (i.e., commuting Hermitian operators) gives the possible results of a non-degenerate measurement. The eigenvectors that generate those one-dimensional blocks of the join are characterized by the ordered $n$-tuples $(\lambda, \ldots, \mu)$ of eigenvalues so the eigenvectors are usually denoted as the eigenkets $|\lambda, \ldots, \mu\rangle$ in the Dirac notation. These Complete Sets of Commuting Operators are Dirac's CSCOs [10] (which are the vector space version of our previous CSCAs).

Since the eigen-alternatives determined by an operator, i.e., eigenvectors, can be obtained by the complete partition joins defined by a CSCO, one might ask if the eigen-alternatives determined by a group representation, i.e., the irreps and their irreducible carrier spaces, could also be obtained by the partition joins defined by some CSCO. Jin-Quan Chen and his colleagues in the Nanjing School have developed a little-known CSCO method to systematically find the irreducible basis vectors for the irreducible spaces that works not only for all representations of finite groups but for all compact Lie groups as needed in QM $([7,[8])$. "[T]he foundation of the new approach is precisely the theory of the complete set of commuting operators (CSCO) initiated by Dirac..." [8, p. 2] Thus the linearized partition joins of the CSCO method extends also to all compact group representations to characterize the maximally definite eigen-alternatives.

The partitional mathematics for sets and vector spaces is summarized in the following table.

\begin{tabular}{|l|l|l|}
\cline { 2 - 3 } \multicolumn{1}{l|}{ Lifting Summary } & Set concept & Vector space concept \\
\hline Partition & $\begin{array}{l}\text { Direct sum decomposition } \pi= \\
\{\mathrm{B}\} \text { of } \mathrm{U}: \mathrm{U}=\uplus \mathrm{B}\end{array}$ & $\begin{array}{l}\text { Direct sum decomposition }\left\{\mathrm{W}_{\mathrm{i}}\right\} \\
\text { of } \mathrm{V}: \mathrm{V}=\sum \oplus \mathrm{W}_{\mathrm{i}}\end{array}$ \\
\hline $\begin{array}{l}\text { Real-valued } \\
\text { Attribute }\end{array}$ & Function $\mathrm{f} \mathrm{U} \rightarrow \mathbb{R}$ & Hermitian operator $\mathrm{L}: \mathrm{V} \rightarrow \mathrm{V}$ \\
\hline $\begin{array}{l}\text { Partition of } \\
\text { attribute }\end{array}$ & $\begin{array}{l}\text { Inverse-image partition } \\
\left\{\mathrm{f}^{-1}(\mathrm{r})\right\} \text { for } \mathrm{f}: \mathrm{U} \rightarrow \mathbb{R}\end{array}$ & $\begin{array}{l}\text { Eigenspace partition } \mathrm{W}_{\mathrm{L}}= \\
\left\{\mathrm{W}_{\lambda}\right\} \text { for } \mathrm{L}: \mathrm{V} \rightarrow \mathrm{V}\end{array}$ \\
\hline $\begin{array}{l}\text { Compatible } \\
\text { partitions }\end{array}$ & Partitions $\pi, \sigma$ on same set $\mathrm{U}$ & $\begin{array}{l}\text { Vector space partitions }\left\{\mathrm{W}_{\mathrm{i}}\right\} \\
\text { and }\left\{\mathrm{X}_{\mathrm{j}}\right\} \text { with common basis }\end{array}$ \\
\hline $\begin{array}{l}\text { Compatible } \\
\text { attributes }\end{array}$ & $\begin{array}{l}\text { Attributes } \mathrm{f}, \mathrm{g}: \mathrm{U} \rightarrow \mathbb{R} \text { defined } \\
\text { on same set } \mathrm{U}\end{array}$ & $\begin{array}{l}\text { Commuting operators } \mathrm{LM}= \\
\mathrm{ML}, \text { i.e., common basis of } \\
\text { simultaneous eigenvectors. }\end{array}$ \\
\hline $\begin{array}{l}\text { Join of compatible } \\
\text { attribute partitions }\end{array}$ & $\begin{array}{l}\mathrm{f}^{-1} \vee \mathrm{g}^{-1}=\left\{\mathrm{f}^{-1}(\mathrm{r}) \cap \mathrm{g}^{-1}(\mathrm{~s})\right\} \text { for } \\
\mathrm{f}, \mathrm{g}: \mathrm{U} \rightarrow \mathbb{R}\end{array}$ & $\begin{array}{l}\mathrm{W}_{\mathrm{L}} \vee \mathrm{W}_{\mathrm{M}}=\left\{\mathrm{W}_{\lambda} \cap \mathrm{W}_{\mu}\right\} \text { for } \mathrm{LM}= \\
\mathrm{ML}\end{array}$ \\
\hline CSCO & $\begin{array}{l}\text { Singleton blocks of } \vee \mathrm{f}_{\mathrm{i}}^{-1} \text { for } \\
\text { compatible attributes }\left\{\mathrm{f}_{\mathrm{i}}^{-1}\right\}\end{array}$ & $\begin{array}{l}\text { One-dim. blocks of } \vee \mathrm{W}_{\mathrm{L}_{\mathrm{i}}} \text { for } \\
\text { commuting operators }\left\{\mathrm{L}_{\mathrm{i}}\right\}\end{array}$ \\
\hline
\end{tabular}

\footnotetext{
${ }^{16}$ One must be careful not to assume that the simultaneous eigenvectors are the eigenvectors for the operator $L M=M L$ due to the problem of degeneracy.
} 
Figure 7: Summary of partition concepts for sets and vector spaces

\section{Quantum Mechanics over sets}

\subsection{The pedagogical model of QM over $\mathbb{Z}_{2}$}

In the tradition of toy models for quantum mechanics, Schumacher and Westmoreland 35] have recently investigated models of quantum mechanics over finite fields. One finite field stands out over the rest, $\mathbb{Z}_{2}$, since vectors in a vector space over $\mathbb{Z}_{2}$ have a natural interpretation, namely as sets that are subsets of a universe set. But in any vector space over a finite field, there is no inner product so the first problem in constructing a model of QM in this context is the definition of Dirac's brackets. Which aspects of the usual treatment of the brackets should be retained and which aspects should be dropped?

Schumacher and Westmoreland chose to have their brackets continue to have values in the base field, e.g., $\mathbb{Z}_{2}=\{0,1\}$, so their "theory does not make use of the idea of probability" [35, p. 919] which certainly constrains the relation to QM. Instead, the values of 0 and 1 are respectively interpreted modally as impossible and possible and hence their name of "modal quantum theory." A number of results from full QM carry over to their modal quantum theory, e.g., no-cloning, superdense coding, and teleportation, but without a probability calculus, the connection to full QM is rather limited. And important results such as Bell's Theorem do not carry over; "in the absence of probabilities and expectation values the Bell approach will not work." [35, p. 921]

But all these limitations can be overcome by the different treatment of the brackets based on crossing the sets-to-vector-space bridge in the other direction (essentially using the basis principle in reverse). That yields a full probability calculus for a model of quantum mechanics over sets (QM/sets) using the $\mathbb{Z}_{2}$ base field. QM/sets yields a probability calculus-and it is a familiar calculus, logical probability theory for a finite universe set of outcomes developed by Laplace, Boole, and others. The only difference from that classical calculus is the vector space formulation which allows different (equicardinal) bases or universe sets of outcomes and thus it is the non-commutative version of classical logical finite probability theory. This allows the development of the QM/sets version of QM results such as Bell's Theorem, the indeterminacy principle, double-slit experiments, and much else in the clear and distinct context of finite sets 17

By developing a sets-version of QM, the concepts and relationships of full QM are represented in a pared-down ultra-simple version that can be seen as representing the essential "logic" of QM. It represents the "logic of QM" in that old sense of "logic" as giving the basic essentials of a theory, not in the sense of giving the behavior of propositions in a theory which is the usual "quantum logic" 2 that was, in effect, based on the usual misdescription of Boolean subset logic as the special case of "propositional" logic.

\section{$5.2 \quad$ Vector spaces over $\mathbb{Z}_{2}$}

$\mathrm{QM} /$ sets is said to be "over $\mathbb{Z}_{2}$ " or " over sets" since the power set $\wp(U) \cong \mathbb{Z}_{2}^{|U|}$ (for a finite non-empty universe set $U)$ is a vector space over $\mathbb{Z}_{2}=\{0,1\}$ where the subset addition $S+T$ is the symmetric difference (or inequivalence) of subsets, i.e., $S+T=S \not \equiv T=S \cup T-S \cap T$ for $S, T \subseteq U$. Given a finite universe set $U=\left\{u_{1}, \ldots, u_{n}\right\}$ of cardinality $n$, the $U$-basis in $\wp(U)$ is the set of singletons

\footnotetext{
${ }^{17}$ Since the development of "categorical quantum mechanics" ([1] and [36]), it is known that much of the mathematics of QM can be formally developed in $F d V e c_{K}$, the category of finite-dimensional vector spaces of a field $K$ and linear maps. It is thus tempting to expect that QM/sets will be the special case of $K=\mathbb{Z}_{2}$. But this is not the case for a variety of reasons; the brackets in $\mathrm{QM} /$ sets take values not in $\mathbb{Z}_{2}$ but in the non-negative integers, and the attributes take their "eigenvalues" in the reals, e.g., real-valued random variables. That is how QM/sets turns out to be the non-commutative version of Laplace-Boole logical finite probability theory. That is very different from a formal model of QM where the scalars (e.g., values of brackets and eigenvalues) are in $\mathbb{Z}_{2}$-such as Schumacher and Westmoreland's modal QT over $\mathbb{Z}_{2}[35$.
} 
$\left\{u_{1}\right\},\left\{u_{2}\right\}, \ldots,\left\{u_{n}\right\}$ and a vector in $\wp(U)$ is specified in the $U$-basis by its $\mathbb{Z}_{2}$-valued characteristic function $\chi_{S}: U \rightarrow \mathbb{Z}_{2}$ for an subset $S \subseteq U$ (e.g., a string of $n$ binary numbers). Similarly, a vector $v$ in $\mathbb{C}^{n}$ is specified in terms of an orthonormal basis $\left\{\left|v_{i}\right\rangle\right\}$ by a $\mathbb{C}$-valued function $\langle-\mid v\rangle:\left\{v_{i}\right\} \rightarrow \mathbb{C}$ assigning a complex amplitude $\left\langle v_{i} \mid v\right\rangle$ to each basis vector. One of the key pieces of mathematical machinery in QM, namely the inner product, does not exist in vector spaces over finite fields but brackets can still be defined using $\left\langle\left.\left\{u_{i}\right\}\right|_{U} S\right\rangle=\chi_{S}\left(u_{i}\right)$ (see below) and a norm can be defined to play a similar role in the probability algorithm of QM/sets.

Seeing $\wp(U)$ as the abstract vector space $\mathbb{Z}_{2}^{n}$ allows different bases in which the vectors can be expressed (as well as the basis-free notion of a vector as a ket). Consider the simple case of $U=\{a, b, c\}$ where the $U$-basis is $\{a\},\{b\}$, and $\{c\}$. But the three subsets $\{a, b\},\{b, c\}$, and $\{a, b, c\}$ also form a basis since: $\{a, b\}+\{a, b, c\}=\{c\} ;\{b, c\}+\{c\}=\{b\}$; and $\{a, b\}+\{b\}=\{a\}$. These new basis vectors could be considered as the basis-singletons in another equicardinal universe $U^{\prime}=\left\{a^{\prime}, b^{\prime}, c^{\prime}\right\}$ where $a^{\prime}=\{a, b\}, b^{\prime}=\{b, c\}$, and $c^{\prime}=\{a, b, c\}$.

In the following ket table, each row is a ket of $\mathbb{Z}_{2}^{|U|} \cong \mathbb{Z}_{2}^{3}$ expressed in the $U$-basis, the $U^{\prime}$-basis, and a $U^{\prime \prime}$-basis.

\begin{tabular}{|c|c|c|}
\hline$U=\{a, b, c\}$ & $U^{\prime}=\left\{a^{\prime}, b^{\prime}, c^{\prime}\right\}$ & $U^{\prime \prime}=\left\{a^{\prime \prime}, b^{\prime \prime}, c^{\prime \prime}\right\}$ \\
\hline \hline$\{a, b, c\}$ & $\left\{c^{\prime}\right\}$ & $\left\{a^{\prime \prime}, b^{\prime \prime}, c^{\prime \prime}\right\}$ \\
\hline$\{a, b\}$ & $\left\{a^{\prime}\right\}$ & $\left\{b^{\prime \prime}\right\}$ \\
\hline$\{b, c\}$ & $\left\{b^{\prime}\right\}$ & $\left\{b^{\prime \prime}, c^{\prime \prime}\right\}$ \\
\hline$\{a, c\}$ & $\left\{a^{\prime}, b^{\prime}\right\}$ & $\left\{c^{\prime \prime}\right\}$ \\
\hline$\{a\}$ & $\left\{b^{\prime}, c^{\prime}\right\}$ & $\left\{a^{\prime \prime}\right\}$ \\
\hline$\{b\}$ & $\left\{a^{\prime}, b^{\prime}, c^{\prime}\right\}$ & $\left\{a^{\prime \prime}, b^{\prime \prime}\right\}$ \\
\hline$\{c\}$ & $\left\{a^{\prime}, c^{\prime}\right\}$ & $\left\{a^{\prime \prime}, c^{\prime \prime}\right\}$ \\
\hline$\emptyset$ & $\emptyset$ & $\emptyset$ \\
\hline
\end{tabular}

Vector space isomorphism: $\mathbb{Z}_{2}^{3} \cong \wp(U) \cong \wp\left(U^{\prime}\right) \cong \wp\left(U^{\prime \prime}\right)$ where row $=$ ket.

\subsection{The brackets}

In a Hilbert space, the inner product is used to define the amplitudes $\left\langle v_{i} \mid v\right\rangle$ and the norm $|v|=$ $\sqrt{\langle v \mid v\rangle}$ where the probability algorithm can be formulated using this norm. In a vector space over $\mathbb{Z}_{2}$, the Dirac notation can still be used to define a norm even though there is no inner product. For a singleton basis element $\{u\} \subseteq U$, the bra $\left\langle\left.\{u\}\right|_{U}: \wp(U) \rightarrow \mathbb{R}\right.$ is defined by the bracket:

$$
\left\langle\left.\{u\}\right|_{U} S\right\rangle=\left\{\begin{array}{l}
1 \text { if } u \in S \\
0 \text { if } u \notin S
\end{array}=\chi_{S}(u)\right.
$$

Then $\left\langle\left.\left\{u_{i}\right\}\right|_{U}\left\{u_{j}\right\}\right\rangle=\chi_{\left\{u_{j}\right\}}\left(u_{i}\right)=\chi_{\left\{u_{i}\right\}}\left(u_{j}\right)=\delta_{i j}$ is the set-version of $\left\langle v_{i} \mid v_{j}\right\rangle=\delta_{i j}$ (for an orthonormal basis $\left.\left\{\left|v_{i}\right\rangle\right\}\right)$. Assuming a finite $U$, the bracket linearly extends to the more general form (where $|S|$ is the cardinality of $S$ ):

$$
\left\langle\left. T\right|_{U} S\right\rangle=|T \cap S| \text { for } T, S \subseteq U 18
$$

The basis principle can be run in reverse to transport a vector space concept to sets. Consider an orthonormal basis set $\left\{\left|v_{i}\right\rangle\right\}$ in a finite dimensional Hilbert space. Given two subsets $T, S \subseteq\left\{\left|v_{i}\right\rangle\right\}$ of the basis set, consider the unnormalized superpositions $\psi_{T}=\sum_{\left|v_{i}\right\rangle \in T}\left|v_{i}\right\rangle$ and $\psi_{S}=\sum_{\left|v_{i}\right\rangle \in S}\left|v_{i}\right\rangle$. Then their inner product in the Hilbert space is $\left\langle\psi_{T} \mid \psi_{S}\right\rangle=|T \cap S|$, which transports (crossing the bridge in the other direction) to $\left\langle\left. T\right|_{U} S\right\rangle=|T \cap S|$ for subsets $T, S \subseteq U$ of the $U$-basis of $\mathbb{Z}_{2}^{|U|}$. In both cases, the bracket gives the size of the overlap or indistinctness of the two vectors or sets.

\footnotetext{
${ }^{18}$ Thus $\left\langle\left. T\right|_{U} S\right\rangle=|T \cap S|$ takes values outside the base field of $\mathbb{Z}_{2}$ just like the Hamming distance function $d_{H}(T, S)=|T+S|$ on vector spaces over $\mathbb{Z}_{2}$ in coding theory. 31]
} 


\subsection{Ket-bra resolution}

The ket-bra $|\{u\}\rangle\left\langle\left.\{u\}\right|_{U}\right.$ is the one-dimensional projection operator:

$$
|\{u\}\rangle\left\langle\left.\{u\}\right|_{U}=\{u\} \cap(): \wp(U) \rightarrow \wp(U)\right.
$$

and the ket-bra identity holds as usual:

$$
\sum_{u \in U}|\{u\}\rangle\left\langle\left.\{u\}\right|_{U}=\sum_{u \in U}(\{u\} \cap())=I: \wp(U) \rightarrow \wp(U)\right.
$$

where the summation is the symmetric difference of sets in $\wp(U)$. The overlap $\left\langle\left. T\right|_{U} S\right\rangle$ can be resolved using the ket-bra identity in the same basis: $\left\langle\left. T\right|_{U} S\right\rangle=\sum_{u}\left\langle\left. T\right|_{U}\{u\}\right\rangle\left\langle\left.\{u\}\right|_{U} S\right\rangle$. Similarly a ket $|S\rangle$ for $S \subseteq U$ can be resolved in the $U$-basis;

$$
|S\rangle=\sum_{u \in U}|\{u\}\rangle\left\langle\left.\{u\}\right|_{U} S\right\rangle=\sum_{u \in U}\left\langle\left.\{u\}\right|_{U} S\right\rangle|\{u\}\rangle=\sum_{u \in U}|\{u\} \cap S||\{u\}\rangle
$$

where a subset $S \subseteq U$ is just expressed as the sum of the singletons $\{u\} \subseteq S$. That is ket-bra resolution in sets. The ket $|S\rangle$ is the same as the ket $\left|S^{\prime}\right\rangle$ for some subset $S^{\prime} \subseteq U^{\prime}$ in another $U^{\prime}$ basis, but when the bra $\left\langle\left.\{u\}\right|_{U}\right.$ is applied to the ket $\left.\mid S\right\rangle=\left|S^{\prime}\right\rangle$, then it is the subset $S \subseteq U$, not $S^{\prime} \subseteq U^{\prime}$, that comes outside the ket symbol |\rangle in $\left\langle\left.\{u\}\right|_{U} S\right\rangle=|\{u\} \cap S| 19$

\subsection{The norm}

The $U$-norm $\|S\|_{U}: \wp(U) \rightarrow \mathbb{R}$ is defined, as usual, as the square root of the bracket 20

$$
\|S\|_{U}=\sqrt{\left\langle\left. S\right|_{U} S\right\rangle}=\sqrt{|S|}
$$

for $S \in \wp(U)$ which is the set-version of the norm $|\psi|=\sqrt{\langle\psi \mid \psi\rangle}$. Note that a ket has to be expressed in the $U$-basis to apply the norm definition so in the above example, $\left\|\left\{a^{\prime}\right\}\right\|_{U}=\sqrt{2}$ since $\left\{a^{\prime}\right\}=\{a, b\}$ in the $U$-basis.

\subsection{The Born Rule}

For a specific basis $\left\{\left|v_{i}\right\rangle\right\}$ and for any nonzero vector $v$ in a finite dimensional complex vector space, $|v|^{2}=\sum_{i}\left\langle v_{i} \mid v\right\rangle\left\langle v_{i} \mid v\right\rangle^{*}$ (* is complex conjugation) whose set version would be: $\|S\|_{U}^{2}=$ $\sum_{u \in U}\left\langle\left.\{u\}\right|_{U} S\right\rangle^{2}$. Since

$$
|v\rangle=\sum_{i}\left\langle v_{i} \mid v\right\rangle\left|v_{i}\right\rangle \text { and }|S\rangle=\sum_{u \in U}\left\langle\left.\{u\}\right|_{U} S\right\rangle|\{u\}\rangle,
$$

applying the Born Rule by squaring the coefficients $\left\langle v_{i} \mid v\right\rangle$ and $\left\langle\left.\{u\}\right|_{U} S\right\rangle$ (and normalizing) gives the probability sums for the eigen-elements $v_{i}$ or $\{u\}$ given a state $v$ or $S$ respectively in QM and QM/sets:

$$
\sum_{i} \frac{\left\langle v_{i} \mid v\right\rangle\left\langle v_{i} \mid v\right\rangle^{*}}{|v|^{2}}=1 \text { and } \sum_{u} \frac{\left\langle\left.\{u\}\right|_{U} S\right\rangle^{2}}{\|S\|_{U}^{2}}=\sum_{u} \frac{|\{u\} \cap S|}{|S|}=1
$$

where $\frac{\left\langle v_{i} \mid v\right\rangle\left\langle v_{i} \mid v\right\rangle^{*}}{|v|^{2}}$ is a 'mysterious' quantum probability while $\frac{\langle\{u\} \mid U S\rangle^{2}}{\|S\|_{U}^{2}}=\frac{|\{u\} \cap S|}{S \mid}$ is the unmysterious Laplacian equal probability $\operatorname{Pr}(\{u\} \mid S)$ rule for getting $u$ when sampling $S 21$

\footnotetext{
${ }^{19}$ The term " $\{u\} \cap S^{\prime \prime}$ is not even defined since it is the intersection of subsets of two different universes.

${ }^{20}$ We use the double-line notation $\|S\|_{U}$ for the $U$-norm of a set to distinguish it from the single-line notation $|S|$ for the cardinality of a set, whereas the customary absolute value notation for the norm of a vector in full QM is $|v|$.

${ }^{21}$ Note that there is no notion of a normalized vector in a vector space over $\mathbb{Z}_{2}$ (another consequence of the lack of an inner product). The normalization is, as it were, postponed to the probability algorithm which is computed in the rationals.
} 


\subsection{Spectral decomposition on sets}

An observable, i.e., a Hermitian operator, on a Hilbert space has a home basis set of orthonormal eigenvectors. In a similar manner, a real-valued attribute $f: U \rightarrow \mathbb{R}$ defined on $U$ has the $U$ basis as its "home basis set." The connection between the numerical attributes $f: U \rightarrow \mathbb{R}$ of QM/sets and the Hermitian operators of full QM can be established by "seeing" the function $f$ as a formal operator: $f \uparrow(): \wp(U) \rightarrow \wp(U)$. Applied to the basis elements $\{u\} \subseteq U$, we may write $f \nmid\{u\}=f(u)\{u\}=r\{u\}$ as the set-version of an eigenvalue equation applied to an eigenvector, where the multiplication $r\{u\}$ is only formal (read $r\{u\}$ as the instruction: give $f$ the value $r$ on $\{u\})$. Then for any subset $S \subseteq f^{-1}(r)$ where $f$ is constant, we may also formally write: $f\lceil S=r S$ as an eigenvalue equation satisfied by all the eigen-sets or eigenvectors $S$ in the eigenspace $\wp\left(f^{-1}(r)\right)$, a subspace of $\wp(U)$, for the eigenvalue $r$. The eigenspaces $\wp\left(f^{-1}(r)\right)$ give a direct sum decomposition (i.e., a vector-space partition) of the whole space $\wp(U)=\sum_{r} \oplus \wp\left(f^{-1}(r)\right)$, just as the set partition $f^{-1}=\left\{f^{-1}(r)\right\}_{r}$ gives a direct sum decomposition of the set $U=\biguplus_{r} f^{-1}(r)$. Since $f^{-1}(r) \cap(): \wp(U) \rightarrow \wp(U)$ is the projection operator 22 to the eigenspace $\wp\left(f^{-1}(r)\right)$ for the eigenvalue $r$, we have the spectral decomposition of a $U$-attribute $f: U \rightarrow \mathbb{R}$ in $\mathrm{QM} /$ sets analogous to the spectral decomposition of a Hermitian operator $L=\sum_{\lambda} \lambda P_{\lambda}$ in QM:

$$
\begin{gathered}
f \uparrow()=\sum_{r} r\left[f^{-1}(r) \cap()\right]: \wp(U) \rightarrow \wp(U) \\
L=\sum_{\lambda} \lambda P_{\lambda}: V \rightarrow V
\end{gathered}
$$

Spectral decomposition of operators in QM/sets and in QM.

\subsection{Lifting and internalization}

QM/sets is a pedagogical model where the internal workings of QM math are laid out in a simplified and "externalized" form. Think of a simplified laboratory model of a complex machine where the parts are visible and easily laid out to clarify the workings of the actual machine. To recover QM math from QM/sets, the base field is lifted from the sets-case of $\mathbb{Z}_{2}$ to QM-case of $\mathbb{C}$, and the externalized forms become internalized (or "encoded") within the vector space. The formal multiplication $r\left[f^{-1}(r) \cap()\right]$ is internalized as an actual multiplication of a scalar times an operator $\lambda P_{\lambda}$ on a vector space over $\mathbb{C}$. The operator representation $L=\sum_{\lambda} \lambda P_{\lambda}$ of an observable is just the lifted internalization or encoding of a numerical attribute $\sum_{r} r\left[f^{-1}(r) \cap()\right]$ made possible by the enriched base field $\mathbb{C}$. The set brackets $\left\langle\left. S\right|_{U} T\right\rangle$ taking values outside the base field $\mathbb{Z}_{2}$ become internalized as an inner product with the same enrichment of the base field to $\mathbb{C}$, and similarly for the $U$-norm that is the square root of the brackets 23

It is the comparative poverty of the base field $\mathbb{Z}_{2}$ that requires the QM/sets brackets and norm to take the externalized values outside the base field and for a formal multiplication to be used in the "operator" representation $f \uparrow()=\sum_{r} r\left[f^{-1}(r) \cap()\right]$ of a numerical attribute $f: U \rightarrow \mathbb{R}$. The only numerical attributes that can be internally represented in $\wp(U) \cong \mathbb{Z}_{2}^{|U|}$ are the 0,1 -attributes or characteristic functions $\chi_{S}: U \rightarrow \mathbb{Z}_{2}$ that are internally represented in the $U$-basis as the projection operators $S \cap(): \wp(U) \rightarrow \wp(U)$.

In the engineering literature, eigenvalues are seen as "stretching or shrinking factors" but that is not their role in QM. The whole machinery of eigenvectors [e.g., the eigen-sets $f \uparrow S=r S$ for $S \subseteq f^{-1}(r)$ in sets], eigenspaces [e.g., the space of all eigen-sets $\wp\left(f^{-1}(r)\right)$ ], and eigenvalues [e.g., $f(u)=r$ ] in full QM is a way of lifting and internalizing or encoding numerical attributes [e.g., $f: U \rightarrow \mathbb{R}$ in the set case] inside a vector space that has a rich enough base field. The old question "Why do attributes in classical physics (like the position or momentum of a particle)

\footnotetext{
${ }^{22}$ Since $\wp(U)$ is now interpreted as a vector space, it should be noted that the projection operator $T \cap(): \wp(U) \rightarrow$ $\wp(U)$ is not only idempotent but linear, i.e., $\left(T \cap S_{1}\right)+\left(T \cap S_{2}\right)=T \cap\left(S_{1}+S_{2}\right)$. Indeed, this is the distributive law when $\wp(U)$ is interpreted as a Boolean ring.

${ }^{23}$ The Schumacher-Westmoreland decision to try to develop quantum theory over $\mathbb{Z}_{2}$ with the brackets taking values in the base field $\mathbb{Z}_{2}$ [35] can thus be seen as an example of premature internalization.
} 
become operators in QM?" is addressed by internalization. The observable operators of QM are the lifted vector space internalizations or encodings over $\mathbb{C}$ of the concept of real-valued attributes (or random variables) on sets.

Moreover, for the internalization of attributes as operators to always be possible, the secular equations for eigenvalues have to have a complete set of solutions so the base field has to be algebraically closed-which addresses another old question of why full QM has the complex numbers $\mathbb{C}$ as its base field.

\subsection{Completeness and orthogonality of projection operators}

The usual completeness and orthogonality conditions on eigenspaces also have set-versions in QM over $\mathbb{Z}_{2}$ :

1. completeness: $\sum_{\lambda} P_{\lambda}=I: V \rightarrow V$ has the set-version: $\sum_{r} f^{-1}(r) \cap()=I: \wp(U) \rightarrow \wp(U)$, and

2. orthogonality: for $\lambda \neq \lambda^{\prime}, P_{\lambda} P_{\lambda^{\prime}}=0: V \rightarrow V$ (where 0 is the zero operator) has the set-version: for $r \neq r^{\prime},\left[f^{-1}(r) \cap()\right]\left[f^{-1}\left(r^{\prime}\right) \cap()\right]=\emptyset \cap(): \wp(U) \rightarrow \wp(U)$.

Note that in spite of the lack of an inner product, the orthogonality of projection operators $S \cap()$ is perfectly well defined in QM/sets where it boils down to the disjointness of subsets, i.e., the cardinality of their overlap (instead of their inner product) being 0 .

\subsection{Pythagorean Theorem for sets}

An orthogonal decomposition of a finite set $U$ is just a partition $\pi=\{B\}$ of $U$ since the blocks $B, B^{\prime}, \ldots$ are orthogonal (i.e., disjoint) and their sum is $U$. Given such an orthogonal decomposition of $U$, we have the:

$$
\|U\|_{U}^{2}=\sum_{B \in \pi}\|B\|_{U}^{2}
$$

Pythagorean Theorem

for orthogonal decompositions of sets.

\subsection{Whence the Born Rule?}

Another old question is: "why the squaring of amplitudes in QM?" A state objectively indefinite between certain definite orthogonal alternatives $A$ and $B$, where the latter are represented by vectors $\vec{A}$ and $\vec{B}$, is represented by the vector sum $\vec{C}=\vec{A}+\vec{B}$. But what is the "strength," "intensity," or relative importance of the vectors $\vec{A}$ and $\vec{B}$ in the vector sum $\vec{C}$ ? That question requires a scalar measure of strength or intensity. The magnitude given by the norm does not answer the question since $\|\vec{A}\|+\|\vec{B}\| \neq\|\vec{C}\|$. But the Pythagorean Theorem shows that the norm-squared gives the scalar measure of "intensity" that answers the question: $\|\vec{A}\|^{2}+\|\vec{B}\|^{2}=\|\vec{C}\|^{2}$ in vector spaces over $\mathbb{Z}_{2}$ or over $\mathbb{C}$. And when the objectively indefinite superposition state is decohered by a distinctionmaking measurement, then the objective probability that the indefinite state will reduce to one of the definite alternatives is given by that objective relative scalar measure of the eigen-alternative's "strength," "intensity," or importance in the indefinite state-and that is the Born Rule. In a slogan, Born is the son of Pythagoras. 


\subsection{Measurement in QM/sets}

The Pythagorean results (for the complete and orthogonal projection operators):

$$
|v|^{2}=\sum_{\lambda}\left|P_{\lambda}(v)\right|^{2} \text { and }\|S\|_{U}^{2}=\sum_{r}\left\|f^{-1}(r) \cap S\right\|_{U}^{2},
$$

give the probabilities for measuring attributes. Since by the Pythagorean Theorem:

$$
|S|=\|S\|_{U}^{2}=\sum_{r}\left\|f^{-1}(r) \cap S\right\|_{U}^{2}=\sum_{r}\left|f^{-1}(r) \cap S\right|,
$$

we have in full QM and in QM/sets:

$$
\sum_{\lambda} \frac{\left|P_{\lambda}(v)\right|^{2}}{|v|^{2}}=1 \text { and } \sum_{r} \frac{\left\|f^{-1}(r) \cap S\right\|_{U}^{2}}{\|S\|_{U}^{2}}=\sum_{r} \frac{\left|f^{-1}(r) \cap S\right|}{|S|}=1 .
$$

Here $\frac{\left|P_{\lambda}(v)\right|^{2}}{|v|^{2}}$ is the mysterious quantum probability of getting $\lambda$ in an $L$-measurement of $v$ while $\frac{\left|f^{-1}(r) \cap S\right|}{|S|}$ has the rather unmysterious interpretation in the pedagogical model, QM/sets, as the probability $\operatorname{Pr}(r \mid S)$ of the random variable $f: U \rightarrow \mathbb{R}$ having the eigen-value $r$ when sampling $S \subseteq U$. Thus the set-version of the Born Rule is not some weird quantum notion of probability on sets but the perfectly ordinary Laplace-Boole rule for the conditional probability $\operatorname{Pr}(r \mid S)=\frac{\left|f^{-1}(r) \cap S\right|}{|S|}$, given $S \subseteq U$, of a random variable $f: U \rightarrow \mathbb{R}$ having the value $r$.

The collecting-together of some eigen-elements $\{u\} \subseteq U$ into a subset $S \subseteq U$ to form an "indefinite element" $S$ has the vector sum $|S\rangle=\sum_{u \in U}\left\langle\left.\{u\}\right|_{U} S\right\rangle|\{u\}\rangle$ in the vector space $\wp(U)$ over $\mathbb{Z}_{2}$ giving the superposition version of the indefinite element. This cements the interpretation of collecting together in sets as superposition in vector spaces.

The indefinite element $S$ is being measured using the observable $f$ where the probability $\operatorname{Pr}(r \mid S)$ of getting the eigenvalue $r$ is $\frac{\left\|f^{-1}(r) \cap S\right\|_{U}^{2}}{\|S\|_{U}^{2}}=\frac{\left|f^{-1}(r) \cap S\right|}{|S|}$ and where the "damned quantum jump" (Schrödinger) goes from $S$ by the projection operator $f^{-1}(r) \cap()$ to the projected resultant state $f^{-1}(r) \cap S$ which is in the eigenspace $\wp\left(f^{-1}(r)\right)$ for that eigenvalue $r$.

The partition operation in QM/sets that describes measurement is the partition join of the partition $\left\{S, S^{c}\right\}$ and $f^{-1}=\left\{f^{-1}(r)\right\}$ so that the initial pure state $S$ (as a mini-blob) is refined into the mixture $\left\{f^{-1}(r) \cap S\right\}$ of possible resultant states. The other states $\left\{f^{-1}(r) \cap S^{c}\right\}$ in the join $f^{-1} \vee\left\{S, S^{c}\right\}$ are not possible or "potential" states starting from $S$. The state resulting from the measurement represents a more-definite element $f^{-1}(r) \cap S$ that now has the definite $f$-value of $r$-so a second measurement would yield the same eigenvalue $r$ with probability $\operatorname{Pr}\left(r \mid f^{-1}(r) \cap S\right)=$ $\frac{\left|f^{-1}(r) \cap\left[f^{-1}(r) \cap S\right]\right|}{\left|f^{-1}(r) \cap S\right|}=\frac{\left|f^{-1}(r) \cap S\right|}{\left|f^{-1}(r) \cap S\right|}=1$ and the same vector $f^{-1}(r) \cap\left[f^{-1}(r) \cap S\right]=f^{-1}(r) \cap S$ using the idempotency of the set-version of projection operators.

This is all just the QM over sets version of the treatment of measurement in standard Dirac-vonNeumann QM where the probability calculus of logical probability theory is lifted and internalized by the Born Rule and where the real attributes or real random variables $f: U \rightarrow \mathbb{R}$ are internalized as observable (Hermitian) operators 24

\subsection{Summary of QM/sets}

These set-versions are summarized in the following table for a finite $U$ and a finite dimensional Hilbert space $V$ with $\left\{\left|v_{i}\right\rangle\right\}$ as any orthonormal basis.

\footnotetext{
${ }^{24}$ See [14] and [15] for a more extensive treatment of measurement using density matrices in both full QM and $\mathrm{QM} /$ sets.
} 


\begin{tabular}{|c|c|}
\hline Vector space over $\mathbb{Z}_{2}:$ QM/sets & Hilbert space case: QM over $\mathbb{C}$ \\
\hline Projections: $S \cap(): \wp(U) \rightarrow \wp(U)$ & $P: V \rightarrow V$ \\
\hline Spectral Decomp.: $f\left\lceil()=\sum_{r} r\left(f^{-1}(r) \cap()\right)\right.$ & $L=\sum_{\lambda} \lambda P_{\lambda}$ \\
\hline Compl.: $\sum_{r} f^{-1}(r) \cap()=I: \wp(U) \rightarrow \wp(U)$ & $\sum_{\lambda} P_{\lambda}=I$ \\
\hline Orthog.: $r \neq r^{\prime},\left[f^{-1}(r) \cap()\right]\left[f^{-1}\left(r^{\prime}\right) \cap()\right]=\emptyset \cap()$ & $\lambda \neq \lambda^{\prime}, P_{\lambda} P_{\lambda^{\prime}}=0$ \\
\hline Brackets: $\left\langle\left. S\right|_{U} T\right\rangle=|S \cap T|=$ overlap of $S, T \subseteq U$ & $\langle\psi \mid \varphi\rangle=$ overlap of $\psi$ and $\varphi$ \\
\hline Ket-bra: $\sum_{u \in U}|\{u\}\rangle\left\langle\left.\{u\}\right|_{U}=\sum_{u \in U}(\{u\} \cap())=I\right.$ & $\sum_{i}\left|v_{i}\right\rangle\left\langle v_{i}\right|=I$ \\
\hline Resolution: $\left\langle\left. S\right|_{U} T\right\rangle=\sum_{u}\left\langle\left. S\right|_{U}\{u\}\right\rangle\left\langle\left.\{u\}\right|_{U} T\right\rangle$ & $\langle\psi \mid \varphi\rangle=\sum_{i}\left\langle\psi \mid v_{i}\right\rangle\left\langle v_{i} \mid \varphi\right\rangle$ \\
\hline Norm: $\|S\|_{U}=\sqrt{\left\langle\left. S\right|_{U} S\right\rangle}=\sqrt{|S|}$ where $S \subseteq U$ & $|\psi|=\sqrt{\langle\psi \mid \psi\rangle}$ \\
\hline Pythagoras: $\|S\|_{U}^{2}=\sum_{u \in U}\left\langle\left.\{u\}\right|_{U} S\right\rangle^{2}=|S|$ & $|\psi|^{2}=\sum_{i}\left\langle v_{i} \mid \psi\right\rangle^{*}\left\langle v_{i} \mid \psi\right\rangle$ \\
\hline Laplace: $S \neq \emptyset, \sum_{u \in U} \frac{\left\langle\left.\{u\}\right|_{U} S\right\rangle^{2}}{\|S\|_{U}^{2}}=\sum_{u \in S} \frac{1}{|S|}=1$ & $|\psi\rangle \neq 0, \sum_{i} \frac{\left\langle v_{i} \mid \psi\right\rangle^{*}\left\langle v_{i} \mid \psi\right\rangle}{|\psi|^{2}}=\frac{\left|\left\langle v_{i} \mid \psi\right\rangle\right|^{2}}{|\psi|^{2}}=1$ \\
\hline Born: $|S\rangle=\sum_{u \in U}\left\langle\left.\{u\}\right|_{U} S\right\rangle|\{u\}\rangle, \operatorname{Pr}(\{u\} \mid S)=\frac{\left\langle\left.\{u\}\right|_{U} S\right\rangle^{2}}{\|S\|_{U}^{2}}$ & $|\psi\rangle=\sum_{i}\left\langle v_{i} \mid \psi\right\rangle\left|v_{i}\right\rangle, \operatorname{Pr}\left(v_{i} \mid \psi\right)=\frac{\left|\left\langle v_{i} \mid \psi\right\rangle\right|^{2}}{|\psi|^{2}}$ \\
\hline$\|S\|_{U}^{2}=\sum_{r}\left\|f^{-1}(r) \cap S\right\|_{U}^{2}=\sum_{r}\left|f^{-1}(r) \cap S\right|=|S|$ & $|\psi|^{2}=\sum_{\lambda}\left|P_{\lambda}(\psi)\right|^{2}$ \\
\hline$S \neq \emptyset, \sum_{r} \frac{\left\|f^{-1}(r) \cap S\right\|_{U}^{2}}{\|S\|_{U}^{2}}=\sum_{r} \frac{\left|f^{-1}(r) \cap S\right|}{|S|}=1$ & $|\psi\rangle \neq 0, \sum_{\lambda} \frac{\left|P_{\lambda}(\psi)\right|^{2}}{|\psi|^{2}}=1$ \\
\hline Measurement: $\operatorname{Pr}(r \mid S)=\frac{\left\|f^{-1}(r) \cap S\right\|_{U}^{2}}{\|S\|_{U}^{2}}=\frac{\left|f^{-1}(r) \cap S\right|}{|S|}$ & $\operatorname{Pr}(\lambda \mid \psi)=\frac{\left|P_{\lambda}(\psi)\right|^{2}}{|\psi|^{2}}$ \\
\hline
\end{tabular}

Probability mathematics for QM over $\mathbb{Z}_{2}$ and for QM over $\mathbb{C}$

Since the probability calculus on the left-side of the table is a non-commutative version of the old Laplace-Boole logical finite probability theory [3], the correspondence with the right-side of the table gives precision to the thesis that the mathematics of QM is a type of logical probability calculus complexified in Hilbert spaces. For instance, the equiprobability or indifference principle of that classical logical finite probability calculus-that is, the equality of all non-zero coefficients in a vector over $\mathbb{Z}_{2}$-generalizes to the QM probability calculus using the coefficients of vectors over $\mathbb{C}$, and the Born Rule applies to orthogonal decompositions in both cases. Crossing the sets-vector-space bridge the other way, we have seen that the classical logical probability calculus (in its non-commutative version) is a type of quantum mechanics over sets.

\subsection{Whence von Neumann's Type 1 and Type 2 processes?}

When some particularly mysterious process like measurement can be clearly and distinctly modeled in QM/sets, then it casts some sense-making light back on full QM. A good example is von Neumann's distinction between Type 1 measurement-like processes and Type 2 processes of unitary evolution 42. In QM/sets, we have seen that measurement is a distinction-making process described by the partition join operation. In terms of the lattice of set partitions, such a "Type 1" process moves up in the lattice to more refined partitions 25 This means in QM/sets that a "Type 2" evolution would be a distinction-preserving process that, as it were, moves horizontally in the lattice of partitions.

\footnotetext{
${ }^{25}$ The usual notion of refinement of partitions, i.e., $\pi=\{B\}$ is more (or equally) refined than $\sigma=\{C\}$, denoted $\sigma \preceq \pi$, if for each $B \in \pi$, there is a $C \in \sigma$ such that $B \subseteq C$, is just the inclusion relation on distinctions, i.e., $\sigma \preceq \pi$ iff $\operatorname{dit}(\sigma) \subseteq \operatorname{dit}(\pi)$, so moving up in the ordering means making more distinctions.
} 


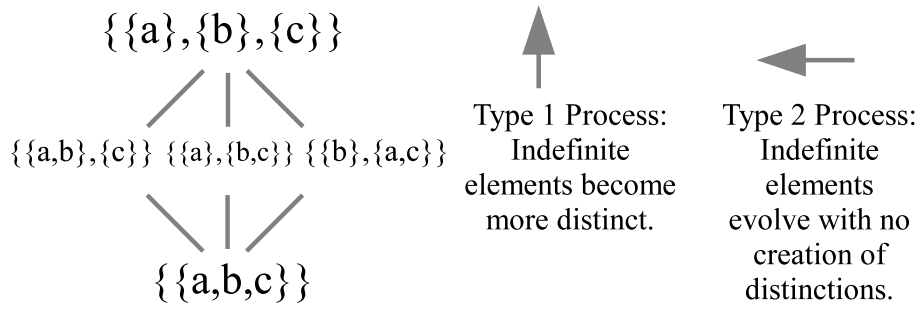

Partition lattice

Figure 8: "Type 1" distinction-making and "Type 2" distinction-preserving processes in QM/sets

A linear transformation $\wp(U) \rightarrow \wp(U)$ that keeps distinct vectors distinct (i.e., preserves distinctions) is just a non-singular transformation 26 This means that a Type 2 process in full QM should be a process that preserves the degree of distinctness and indistinctness. Given two normalized quantum states $\psi$ and $\varphi$, the brackets $\langle\psi \mid \varphi\rangle$ can be interpreted as the degree of indistinctness (or identification) of the states with the extreme values of $\langle\psi \mid \varphi\rangle=1$ for full indistinctness or identification, i.e., $\psi=\varphi$, and $\langle\psi \mid \varphi\rangle=0$ for zero indistinctness, i.e., the full distinctness of orthogonality. Hence under this partitional approach to understanding or making sense of QM, the distinctionpreserving Type 2 processes are the ones that preserve the degree of indistinctness $\langle\psi \mid \varphi\rangle$, i.e., the unitary transformations. Thus by this approach, we can philosophically derive the mathematical form of the indistinctness-preserving transformations in full QM. Moreover, by Stone's Theorem in mathematics, those unitary transformations are given by (the complex exponential of) a Hermitian operator $H$ that would describe how the "substance" changes to preserve indistinctness 27

The partitional follow-the-math approach to understanding QM shows that the mathematical form of full QM is a type of logical probability system expressed in terms of vector spaces rather than sets (the usual setting for logic and the Laplace-Boole logical probability theory). But at some point this mathematical metaphysics has to pass the torch to physics to give empirical content to the formalism. As previously noted, Heisenberg tells us that "substance" is energy, but this "follow the partitional math" approach to making sense out of QM of course cannot derive that physical fact nor the form of the operator $H$ ("H" as in Hamiltonian) for a physical system.

There has been much concern and mystery in the foundations of quantum mechanics about the Type 1 measurement processes-in contrast to the clarity of describing the Type 2 processes as unitary transformations. That is another old question. We have seen that this question can be answered at the mathematical level by the partition join operation. The mathematical description of a measurement in $\mathrm{QM} /$ sets as the join of compatible set-partitions lifts to the mathematical description of a measurement in full QM as the join of compatible vector-space partitions-where one partition describes the distinction-making measurement apparatus representing the observable being measured and the other partition is the indiscrete (i.e., one block) partition representing the given pure state to be measured or, better, to be decohered 28 Using the respective partition join operations, a complete set of compatible attributes (CSCA) gives a non-degenerate measurement in $\mathrm{QM} /$ sets, and a complete set of commuting operators (CSCO) gives a non-degenerate measurement in full QM. Thus the clear and distinct distinction between "Type 1" distinction-making and "Type 2" distinction-preserving processes in the pedagogical model of QM/sets makes sense of the

\footnotetext{
${ }^{26}$ Thus the gates in quantum computing over $\mathbb{Z}_{2}$ are the non-singular linear transformations (35] and 15]).

${ }^{27}$ See the previous Figure 3 on how "substance" is constant or conserved in the partition lattice regardless of whether the changes preserve distinctions ("Type 2" processes-imaged in Figure 8 as lateral moves in the lattice) or create distinctions ("Type 1" processes-imaged as upward moves in the lattice). Also the "Type 1" processes that create distinctions will destroy or wipe out distinctions in another incompatible basis.

${ }^{28}$ See [15] for the development of this using density matrices. This partition mathematical description of the Type 1 measurement process does not give the physical characterization of a distinction-creating measurement apparatus anymore than this approach gives the physical characterization of the distinction-preserving Type 2 unitary processes.
} 
von Neumann Type 1 distinction-making measurements and Type 2 distinction-preserving unitary transformations in full QM.

\section{Final remarks}

There are two meta-physical visions of reality suggested by classical physics (objectively definite reality) and by quantum physics (objectively indefinite reality). The problem of interpreting QM is essentially the problem of making sense out of the notion of objective indefiniteness. Our sensemaking strategy was to follow the lead of the mathematics.

The definiteness of classical physics is associated with the notion of a subset and is logically expressed in the classical Boolean logic of subsets. The indefiniteness of quantum physics is associated with the notion of a quotient set, equivalence relation, or partition, and the corresponding logic is the recently developed logic of partitions [12. Moreover, those associated notions of subsets and quotient sets are category-theoretically dual to one another, so from that viewpoint, those are the only two possible frameworks to describe reality. Common sense and classical physics assumes the objectively definite type of reality, but quantum physics strongly indicates an objectively indefinite reality at the quantum level. Hence our approach to interpreting quantum mechanics is not flights of fantasy (e.g., about many worlds or realms of hidden variables) but is trying to make sense out of objective indefiniteness.

Our sense-making strategy was implemented by developing the mathematics and logic of partitions at the connected conceptual levels of sets and vector spaces. Set concepts are transported to complex vector spaces to yield the mathematical machinery of full QM, and the complex vector space concepts of full QM are transported to the set-like vector spaces over $\mathbb{Z}_{2}$ to yield the rather fulsome pedagogical model of quantum mechanics over sets or QM/sets.

In this manner, we have tried to use partition concepts to make sense of objective indefiniteness and thus to interpret quantum mechanics.

\section{References}

[1] Abramsky, Samson and Bob Coecke 2004. A categorical semantics of quantum protocols. In Proceedings of the 19th IEEE Symposium on Logic in Computer Science (LiCS'04). IEEE Computer Science Press: 415-425.

[2] Birkhoff, Garrett and John von Neumann 1936. The Logic of Quantum Mechanics. Annals of Mathematics. 37 (4): 823-43.

[3] Boole, George 1854. An Investigation of the Laws of Thought on which are founded the Mathematical Theories of Logic and Probabilities. Cambridge: Macmillan and Co.

[4] Brading, Katherine and Harvey R. Brown 2003. Symmetries and Noether's theorems. In Symmetries in Physics: Philosophical Reflections. Katherine Brading and Elena Castellani eds., Cambridge UK: Cambridge University Press: 89-109.

[5] Busch, P. and G. Jaeger 2010. Unsharp Quantum Reality. Foundations of Physics. 40: 13411367.

[6] Castellani, Elena 2003. Symmetry and equivalence. In Symmetries in Physics: Philosophical Reflections. Katherine Brading and Elena Castellani eds., Cambridge UK: Cambridge University Press: 425-436.

[7] Chen, Jin-Quan, Mei-Juan Gao, and Guang-Qun Ma 1985. The representation group and its application to space groups. Reviews of Modern Physics. 57 (1): 211-278. 
[8] Chen, Jin-Quan, Jialun Ping, and Fan Wang 2002. Group Representation Theory for Physicists (2nd ed.). Singapore: World Scientific.

[9] Cohen-Tannoudji, Claude, Bernard Diu, and Franck Laloë 2005. Quantum Mechanics: Volumes 1 and 2. New York: John Wiley \& Sons.

[10] Dirac, P. A. M. 1958. The Principles of Quantum Mechanics (4th ed.). Oxford: Clarendon Press.

[11] Ellerman, David 2009. Counting Distinctions: On the Conceptual Foundations of Shannon's Information Theory. Synthese. 168 (1 May): 119-149. All my papers downloadable at http://www.ellerman.org.

[12] Ellerman, David 2010. The Logic of Partitions: Introduction to the Dual of the Logic of Subsets. Review of Symbolic Logic. 3 (2 June): 287-350.

[13] Ellerman, David 2013. An Introduction to Logical Entropy and its relation to Shannon Entropy. International Journal of Semantic Computing. 7 (2): 121-145.

[14] Ellerman, David 2013. The Objective Indefiniteness Interpretation of Quantum Mechanics, [quant-ph] arXiv:1210.7659.

[15] Ellerman, David 2013. Quantum mechanics over sets. [quant-ph] arXiv:1310.8221.

[16] Ellerman, David 2014. An introduction of partition logic. Logic Journal of the IGPL. 22 (1): 94-125.

[17] Falkenburg, Brigitte 2007. Particle Metaphysics: A Critical Account of Subatomic Reality. Berlin: Springer-Verlag.

[18] Feyerabend, Paul 1983 (orig. 1962). Problems of Microphysics. In Frontiers of Science and Philosophy. Robert G. Colodny ed., Lanham MD: University Press of America: 189-283.

[19] Feynman, Richard P., Robert B. Leighton and Matthew Sands 1965. The Feynman Lectures on Physics: Quantum Mechanics (Vol. III). Reading MA: Addison-Wesley.

[20] Fine, Arthur 1986. The Shaky Game: Einstein, Realism, and the Quantum Theory. Chicago: University of Chicago Press.

[21] French, Steven and Decio Krause 2003. Quantum Vagueness. Erkenntnis. 59: 97-124.

[22] Heisenberg, Werner 1958. Physics $\&$ Philosophy: The Revolution in Modern Science. New York: Harper Torchbooks.

[23] Hoffman, Kenneth and Ray Kunze 1961. Linear Algebra. Englewood Cliffs NJ: Prentice-Hall.

[24] Jaeger, Gregg 2014. Quantum Objects: Non-Local Correlation, Causality and Objective Indefiniteness in the Quantum World. Heidelberg: Springer.

[25] James, William 1952 (1890). The Principles of Psychology (Great Books Series \#53). Chicago: Encyclopedia Britannica.

[26] Jammer, Max 1966. The Conceptual Development of Quantum Mechanics. New York: McGrawHill.

[27] Ladyman, James and Tomasz Bigaj 2010. The Principle of Identity of Indiscernables. and Quantum Mechanics. Philosophy of Science. 77 (January): 117-136.

[28] Lawvere, F. William and Robert Rosebrugh 2003. Sets for Mathematics. Cambridge: Cambridge University Press. 
[29] Lowe, E. J. 1994. Vague Identity and Quantum Indeterminacy. Analysis. 54 (2): 110-114.

[30] Mackey, George W. 1993. The Mathematical Papers. In The Collected Works of Eugene Paul Wigner: Vol. 1. Arthur Wightman ed., Berlin: Springer-Verlag: 241-290.

[31] McEliece, Robert J. 1977. The Theory of Information and Coding: A Mathematical Framework for Communication (Encyclopedia of Mathematics and its Applications, Vol. 3). Reading MA: Addison-Wesley.

[32] McKenzie, Kerry 2013. Priority and Particle Physics: Ontic Structural Realism as a Fundamentality Thesis. British Journal for the Philosophy of Science. Online doi:10.1093/bjps/axt017.

[33] Mittelstaedt, Peter 1998. The Constitution of Objects in Kant's Philosophy and in Modern Physics. In Interpreting Bodies: Classical and Quantum Objects in Modern Physics. Elena Castellani ed., Princeton: Princeton University Press: 168-180.

[34] Roberts, Bryan W. 2011. Group Structural Realism. British Journal for the Philosophy of Science. 62: 47-69.

[35] Schumacher, B. and M. Westmoreland 2012. Modal Quantum Theory. Foundations of Physics, $42,918-925$.

[36] Selinger, Peter 2007. Dagger compact closed categories and completely positive maps. Quantum Programming Languages, Electronic Notices in Theoretical Computer Science. 170: 139-163.

[37] Shimony, Abner 1988. The reality of the quantum world. Scientific American. 258 (1): 46-53.

[38] Shimony, Abner 1989a. Conceptual foundations of quantum mechanics. In The New Physics. Paul Davies ed., Cambridge: Cambridge University Press: 373-395.

[39] Shimony, Abner 1989b. Search for a Worldview Which Can Accommodate Our Knowledge of Microphysics. In Philosophical Consequences of Quantum Theory: Reflections on Bell's Theorem. James T. Cushing and Ernan McMullin eds., Notre Dame, IN: University of Notre Dame Press: 25-37.

[40] Stairs, Allen 1983. Quantum Logic, Realism, and Value Definiteness. Philosophy of Science. 50 (4): $578-602$.

[41] Teller, Paul 1989. Relativity, Relational Holism, and the Bell Inequalities. In Philosophical Consequences of Quantum Theory: Reflections on Bell's Theorem. James T. Cushing and Ernan McMullin eds., Notre Dame, IN: University of Notre Dame Press: 208-223.

[42] von Neumann, John 1955. Mathematical Foundations of Quantum Mechanics. Robert T. Beyer trans., Princeton: Princeton University Press.

[43] Weyl, Hermann 1949. Philosophy of Mathematics and Natural Science. Princeton: Princeton University Press.

[44] Wigner, Eugene P. 1939. On Unitary Representations of the Inhomogeneous Lorentz Group. Annals of Mathematics. 40: 149-204. 